In cooperation with the Texas Natural Resource Conservation Commission

Regional Equations for Estimating Mean Annual and Mean Seasonal Runoff for Natural Basins in Texas, Base Period 1961-90

Water-Resources Investigations Report 00-4064

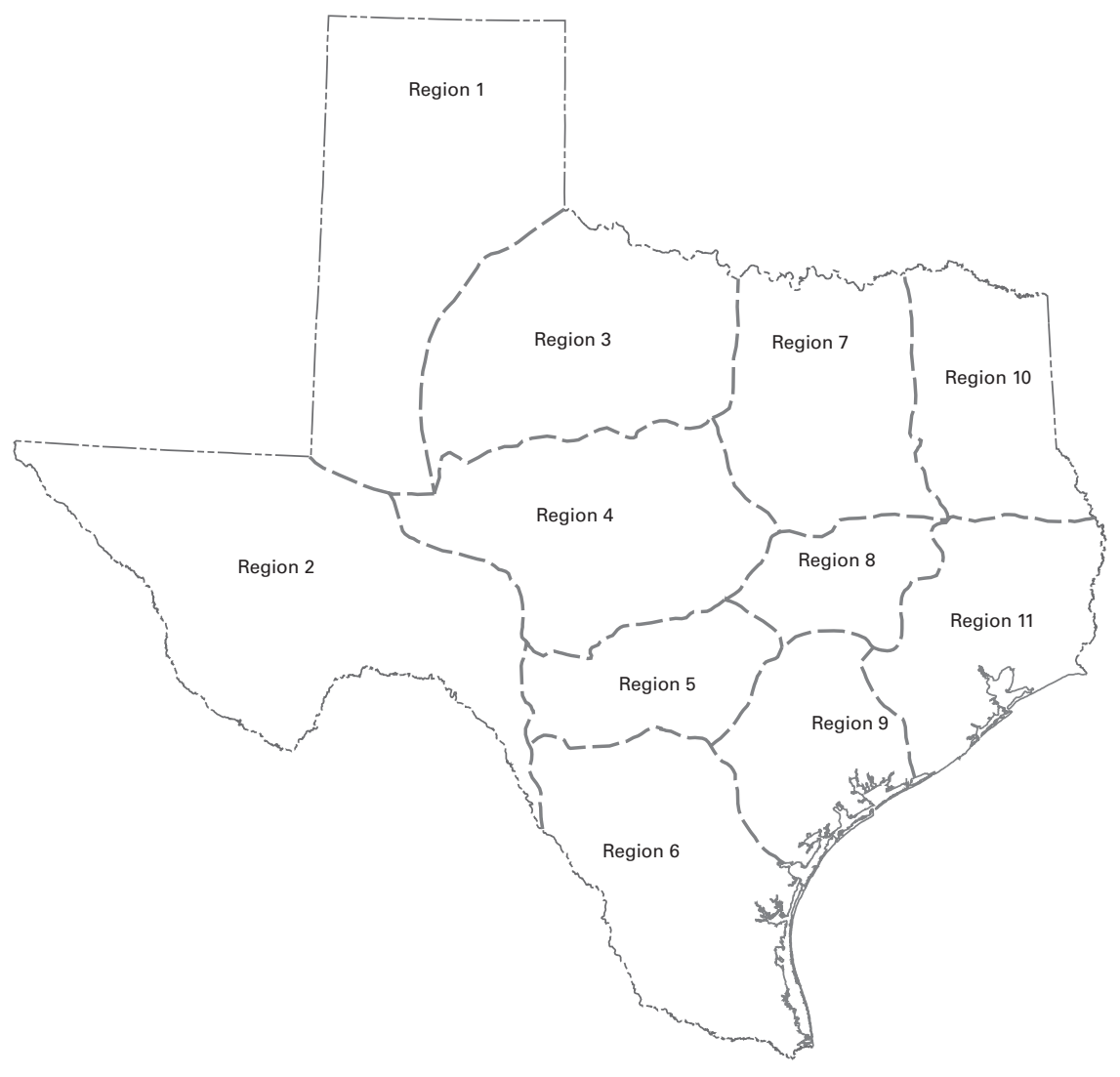

U.S. Department of the Interior

U.S. Geological Survey 
Blank Page 
U.S. Department of the Interior

U.S. Geological Survey

\section{Regional Equations for Estimating Mean Annual and Mean Seasonal Runoff for Natural Basins in Texas, Base Period 1961-90}

By Jennifer Lanning-Rush

\section{U.S. GEOLOGICAL SURVEY}

Water-Resources Investigations Report 00-4064

In cooperation with the Texas Natural Resource Conservation Commission

Austin, Texas

2000 


\section{U.S. DEPARTMENT OF THE INTERIOR}

Bruce Babbitt, Secretary

\section{U.S. GEOLOGICAL SURVEY}

Charles G. Groat, Director

Any use of trade, product, or firm names is for descriptive purposes only and does not imply endorsement by the U.S. Government.

For additional information write to

\section{District Chief}

U.S. Geological Survey

8027 Exchange Dr.

Austin, TX 78754-4733

E-mail: dc_tx@usgs.gov

Copies of this report can be purchased from

U.S. Geological Survey

Branch of Information Services

Box 25286

Denver, CO 80225-0286

E-mail: infoservices@usgs.gov 


\section{CONTENTS}

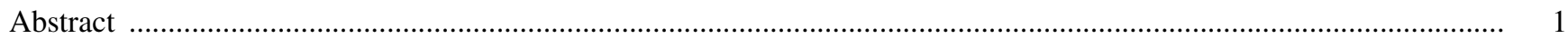

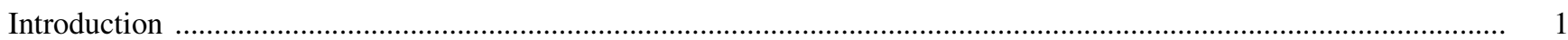

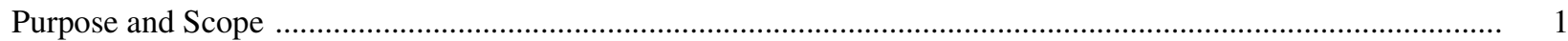

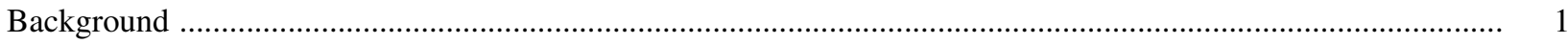

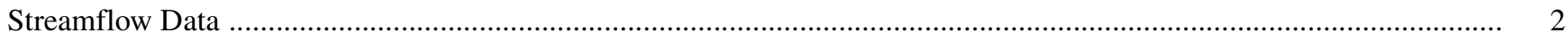

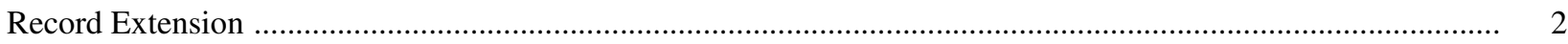

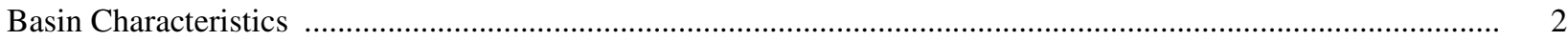

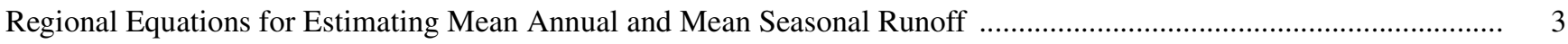

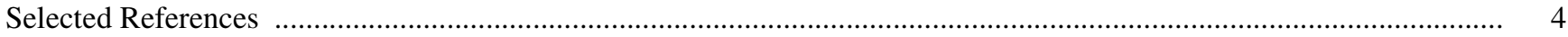

\section{PLATE}

(Plate is in pocket)

1. Map showing location of selected streamflow-gaging stations with natural basins in or near Texas

\section{FIGURES}

1-6. Maps showing:

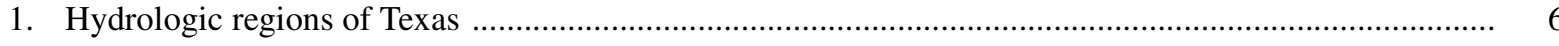

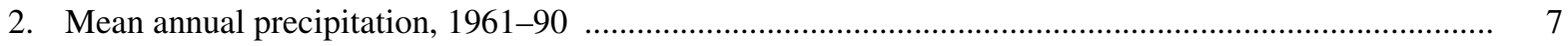

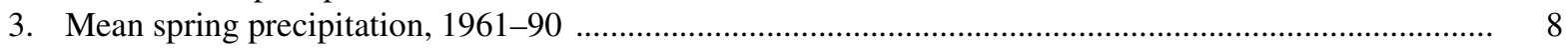

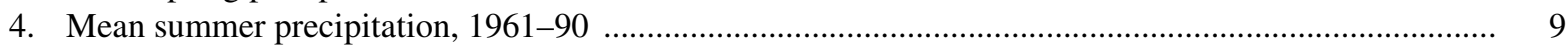

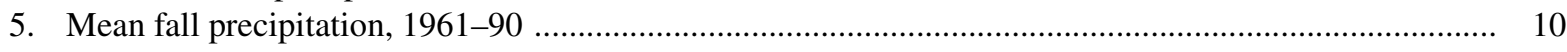

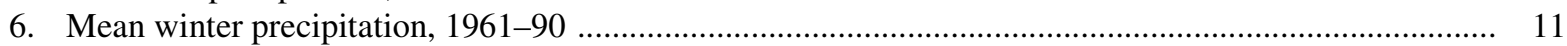

\section{TABLES}

1. Basin characteristics for streamflow-gaging stations in or near Texas, with at least 8 years of mean annual streamflow data, 1961-90

2. Regression equations for estimating mean annual and mean seasonal runoff for regions of Texas 12 26 
Blank Page 


\title{
Regional Equations for Estimating Mean Annual and Mean Seasonal Runoff for Natural Basins in Texas, Base Period 1961-90
}

\author{
By Jennifer Lanning-Rush
}

\begin{abstract}
Regional equations were developed for estimating mean annual and mean seasonal runoff for natural basins in Texas. The equations, which are based on the statistical relation between streamflow and basin characteristics, use streamflow data and basin characteristics from U.S. Geological Survey streamflow-gaging stations within natural basins and with a least 8 years of data during 1961-90. The State was divided into 11 hydrologic regions on the basis of previous studies. The final equations for estimating mean annual and mean seasonal runoff were developed from 228 streamflow-gaging stations. Contributing drainage area and mean annual or mean seasonal precipitation were determined to be the most significant basin characteristics in each region.
\end{abstract}

\section{INTRODUCTION}

The Safe Drinking Water Act Amendments of 1996 (Public Law 104-182) require that each State prepare a source-water assessment for all public water supplies [sections 1453 and 1428(b)]. States are required to determine (1) the drinking-water sources, (2) the origin of both existing and potential contaminants to drinkingwater sources, and (3) the intrinsic susceptibility of drinking-water sources to contamination. The U.S. Geological Survey (USGS), in cooperation with the Texas Natural Resource Conservation Commission (TNRCC), began a study in 1998 to develop a method for estimating mean annual and mean seasonal runoff for water-supply intakes within natural drainage basins in Texas. The results of this study are needed to evaluate and manage the supply of the State's surface-water resources as part of the Texas source-water assessment.

\section{Purpose and Scope}

The purpose of this report is to present and qualify multivariate regional equations to estimate runoff volume for natural basins in Texas. The equations estimate mean annual and mean seasonal runoff on the basis of the statistical relation between streamflow and basin characteristics. The streamflow data and basin characteristics are from USGS continuous-record, streamflow-gaging stations within natural basins and with at least 8 years of data during 1961-90. For this report, a natural basin is defined as a basin with less than 10-percent impervious cover, less than 10-percent of its drainage area controlled by reservoirs, and no other human-related factors that would affect streamflow. Some continuous-record, streamflow-gaging stations were omitted from the study because (1) drainage areas are greater than 10,000 square miles, (2) annual runoff might be affected by reservoirs, (3) substantial amounts of withdrawal or return flow from the basin might occur, (4) streamflow is lost as recharge along the Edwards aquifer, (5) springflow can affect runoff volumes, or (6) records cannot be extended (discussed later in "Record Extension" section). Also included in the study are streamflow-gaging stations in New Mexico and Oklahoma that are less than 25 miles from the Texas border, within a natural basin, and with 30 years of record during 1961-90. The 228 gaging stations that met this criteria are shown on plate 1 .

\section{Background}

Natural runoff varies principally according to temporal and areal variations in precipitation but is also influenced by topography, climate, soil characteristics, geology, and evapotranspiration. Texas is a large region with considerable climatic and physiographic variability. The mean annual precipitation differs little from north to south but differs greatly from west to east. 
Accordingly, runoff can vary tremendously across the State.

Annual precipitation, in this report, represents the calendar year instead of the USGS water year (October 1 to September 30). The seasonality of precipitation was accounted for by developing equations representing each of the four seasons as follows: (1) spring-April, May, and June; (2) summer-July, August, and September; (3) fall-October, November, and December; and (4) winter-January, February, and March.

To account for the areal variability in climate and physiography, the State was divided into regions for this study. Asquith and Slade (1995) delineated hydrologic regions (fig. 1 at end of report) on the basis of areas with similar climatic and physiographic characteristics. To define their regions, Asquith and Slade considered regions presented by Carr (1967), Kier and others (1977), and Schroeder and Massey (1977). The hydrologic regions developed by Asquith and Slade (1995) were considered suitable for this study with some modifications because of insufficient streamflow data in regions 1, 2, 6, and 8 . Regions 1 and 2 were combined for this study because they have similar climates. Streamflow-gaging stations in region 9 that are within 50 miles of the region 6 boundary also were included in analysis for region 6 because of similar physiography. Stations within 30 miles and upstream of region 8 that are in the Trinity and Brazos River Basins also were included in analysis for region 8. Stations used in analysis for more than one hydrologic region are noted in table 1 at end of report.

\section{STREAMFLOW DATA}

The base period 1961-90 was used to coincide with the latest global standard normals period. Thirtyyear periods (1941-70, 1951-80, 1961-90) are standards used by the World Meteorological Organization (WMO) to report climatological standard normals (World Meteorological Organization, 1984). The National Climatic Data Center (NCDC) computes normals for the United States every decade (Guttman, 1989). To further justify use of this base period, an analysis was made for all streamflow stations within natural basins in Texas with at least 50 years of data. The analysis indicates that the 1961-90 period adequately represents average streamflow conditions in Texas.

\section{Record Extension}

If streamflow records for the 30-year period were incomplete for a station (short-term station), the records were extended using a station with streamflow record for that missing period (base station). The line of organic correlation (LOC) (Helsel and Hirsch, 1992) was used to estimate missing records during the 30-year period 1961-90. The LOC is analogous to an ordinary least-squares regression, except that ordinary regression minimizes the squared vertical deviations of the dependent variable (streamflow at short-term station) from the regression line, whereas the LOC technique minimizes the areas of the right triangles formed by the horizontal and vertical deviations from the regression line. The LOC is commonly used and recommended for estimation of missing records (Helsel and Hirsch, 1992).

For each short-term station, a base station was selected that would provide the basis of all the missing data. No base station was used to extend missing record at a short-term station unless the two stations had at least 5 concurrent years of streamflow. Further criteria common to the short-term and base stations included (1) contributing drainage area within 2 log cycles of each other, (2) location within the same hydrologic region or at least within 200 miles of each other, and (3) correlation coefficient of at least 0.5 for mean annual streamflow of each station. The base station was chosen from a set of stations compiled on the basis of linearity of the relation between mean annual streamflow data points for the short-term and base stations. Estimated streamflows for the short-term station are perfectly correlated with the concurrent base station streamflows, but the actual relation between short-term and base stations is highly variable. Therefore, the excluded records will be less reliable than actual gaged records. In an effort to compensate for the fact that the extended record has considerable error, each short-term station used in the final weighted regression analyses (discussed later) was given a weight factor equal to the total number of actual gaged years during 1961-90. Table 1 includes the weight factors assigned to each streamflow-gaging station in the study.

\section{Basin Characteristics}

Six basin characteristics were identified as possible independent variables for regression equation development. The six basin characteristics selected on the basis of availability, probable hydrologic significance, 
and results of related studies (Asquith and Slade, 1995, Raines, 1998, Thomas and Benson, 1970) are:

A-Contributing drainage area (in square miles) is the geographical area enclosed by a topographic divide from which all surface runoff drains into the stream above the gaging station. Data is stored in the Basin Characteristics File of the USGS Water Data Storage and Retrieval System (WATSTORE). (Calculated using the largest-scale USGS topographic maps available, usually 1:24,000.)

$\mathbf{L}$-Stream length (in miles) is the distance measured along the longest mapped channel from the gaging station to the headwaters, based on USGS topographic maps (scale, 1:100,000).

SL-Stream slope (in feet per mile) is the ratio of (1) the change in elevation of the longest mapped channel from the station to the headwaters to (2) the length of the longest mapped channel, based on USGS topographic maps (scale, 1:100,000).

SH-Basin shape factor is the ratio of the square of the stream length $(\mathrm{L})$ to the contributing drainage area (A), which mathematically represents the ratio of the longest mapped channel length to the mean width of the basin.

MAP-Mean annual precipitation on the basin for 1961-90 (in inches), as determined from data published by the NCDC (fig. 2 [at end of report], data from Owenby and Ezell, 1992).

MSP-Mean seasonal precipitation on the basin for 1961-90 (in inches), as determined from data published by the NCDC (figs. 3 to 6 [at end of report], data from Owenby and Ezell, 1992).

\section{REGIONAL EQUATIONS FOR ESTIMATING MEAN ANNUAL AND MEAN SEASONAL RUNOFF}

During multiple-regression analysis, it is important that the various independent variables not be highly correlated with other independent variables. Not adhering to this requirement could yield unstable values for the regression coefficients, which might lead to difficulties in interpreting the effectiveness of various independent variables included in the equation. However, it is not possible to use basin characteristics that are entirely independent of each other because many natural topographic and climatic variables exhibit some degree of interdependence. The data were transformed to common logarithms to produce more linear relations among the dependent and independent variables. A simple cor- relation matrix of the basin characteristics was completed to investigate the amount of interdependence among the variables for each hydrologic region. Several variables indicated relatively high interdependence; some examples include positive correlation between contributing drainage area and main-channel length and between basin shape factor and main-channel length and inverse correlation between contributing drainage area and main-channel slope. Of the highly correlated basin characteristics, those with correlation coefficients greater than 0.8 , only the most significant characteristics were used in the final regression equations for a particular hydrologic region.

The final equations (table 2 at end of report) for estimating mean annual and mean seasonal runoff were developed using weighted least-squares (WLS) regression of streamflow data and basin characteristics from 228 streamflow-gaging stations. In WLS regression, each dependent variable is given a weight representative of its relative accuracy (error). The greater weights are assigned to values that have greater accuracy (less error). The weight factor for each station is the total number of gaged years for the 1961-90 base period. Weight factors in this study range from 8 to 30 . In most of the regression equations, contributing drainage area was the most significant explanatory variable, and mean annual or mean seasonal precipitation was the next most significant variable. The final equations were modified to use the same independent variables within each hydrologic region. In these instances, variables that were significant in most of the equations for any given season were selected as key variables, and the regressions were repeated using the key variables as the only independent variables. The final regression equations developed for each annual and seasonal period, in each region, are listed in table 2 . Also listed in the table are the ranges of independent variables and standard errors associated with each equation.

Regression equations of the following form were derived:

$$
\log \mathrm{Q}_{\mathrm{x}}=\mathrm{a}+\mathrm{b} \log \mathrm{A}+\mathrm{c} \log \mathrm{B}+\ldots \mathrm{n} \log \mathrm{M},
$$

where

$$
\begin{aligned}
\mathrm{Q}_{\mathrm{X}}= & \text { mean runoff, in cubic feet per second, for } \\
& \quad \text { either annual, spring, summer, fall, or } \\
& \text { winter; } \\
\mathrm{a}= & \text { regression constant; } \\
\mathrm{b}, \mathrm{c}, \ldots \mathrm{n}= & \text { regression coefficients; and }
\end{aligned}
$$




\section{$\mathrm{A}, \mathrm{B}, \ldots \mathrm{M}=$ basin characteristics.}

The equations were transformed from logarithm form by taking antilogarithms; the resulting equations have the following form:

$$
\mathrm{Q}_{\mathrm{x}}=10^{\mathrm{a}} \mathrm{A}^{\mathrm{b}} \mathrm{B}^{\mathrm{c}} \ldots \mathrm{M}^{\mathrm{n}}
$$

Independent variables (basin characteristics) were included in the equation analysis if the F statistic ${ }^{1}$ was greater than 5 . The WLS regression also provided statistical measures of the suitability of the equations, such as, standard errors of estimate and coefficients of determination (r-squared). In general, smaller standard errors and larger coefficients of determination result in more reliable equations.

The equations listed in table 2 are based on the range of independent variables associated with the stations in the study, and therefore, the equations might have considerable error for sites with basin characteristics outside of these ranges and should not be used for such sites. Probability plots and residual plots were used to verify each equation in each region. It is preferable that the mean annual runoff equations be used to calculate the mean annual runoff, rather than summing the four estimated mean seasonal runoffs, because the annual equations have less error than the seasonal equations.

\section{SUMMARY}

Regional equations for estimating mean annual and mean seasonal runoff for natural basins are needed for the Texas source-water assessment. The equations are based on the statistical relation between streamflow and basin characteristics. The streamflow data and basin characteristics are from USGS continuous-record, streamflow-gaging stations within natural basins and with a least 8 years of data during 1961-90. The State was divided into 11 hydrologic regions on the basis of previous studies; however, regions 1 and 2 were combined for this study because of their similar climate. Some stations were used in the analysis for more than one region.

\footnotetext{
${ }^{1} \mathrm{~F}$ statistic-A test statistic (ratio of the variation explained by the independent variable to the variation not unexplained). The larger the value of $\mathrm{F}$, the stronger the evidence for rejection of the null hypothesis that the regression coefficient of the independent variable is zero (Ott, 1993).
}

The base period of 1961-90 was used for the streamflow data to coincide with the latest global standard normals period. Records for short-term stations (incomplete for the base period) were extended using records from base stations (complete for the base period). Six basin characteristics were identified as possible independent variables for regression equation development.

The final equations for estimating mean annual and mean seasonal runoff were developed using weighted least-squares regression of streamflow data and basins characteristics from 228 streamflow-gaging stations. The same independent variables were used within each region. Contributing drainage area and mean annual or mean seasonal precipitation were determined to be the most significant basin characteristics in each region.

\section{SELECTED REFERENCES}

Asquith, W.H., and Slade, R.M., Jr., 1995, Documented and potential extreme peak discharges and relation between potential extreme peak discharges and probable maximum flood peak discharges in Texas: U.S. Geological Survey Water-Resources Investigations Report 95-4249, $58 \mathrm{p}$.

Carr, J.T., 1967, The climate and physiography of Texas: Austin, Texas Water Development Board Report 53, $27 \mathrm{p}$.

Christensen, R.C., Johnson, E.B., and Plantz, G.G., 1986, Manual for estimating selected streamflow characteristics of natural-flow streams in the Colorado River Basin in Utah: U.S. Geological Survey Water-Resources Investigations Report 85-4297, 39 p.

Guttman, N.B., 1989, Statistical descriptors of climate: Bulletin of the American Meteorological Society, v. 70, no. 6, p. 602-607.

Hejl, H.R., Jr., 1984, Use of selected basin characteristics to estimate mean annual runoff and peak discharges for ungaged streams in drainage basins containing strippable coal resources, northwestern New Mexico: U.S. Geological Survey Water-Resources Investigations Report 84-4260, $17 \mathrm{p}$.

Helsel, D.R., and Hirsch, R.M., 1992, Studies in environmental science 49-Statistical methods in water resources: Amsterdam, Elsevier, 522 p.

Holtschlag, D.J., and Croskey, H.M., 1984, Statistical models for estimating flow characteristics of Michigan streams: U.S. Geological Survey Water-Resources Investigations Report 84-4207, 80 p.

Kier, R.S., Garner, L.E., and Brown, L.F., 1977, Land resources of Texas: Austin, University of Texas, Bureau of Economic Geology report, $42 \mathrm{p}$. 
Omang, R.J., and Parrett, C., 1984, A method for estimating mean annual runoff of ungaged streams based on basin characteristics in central and eastern Montana: U.S. Geological Survey Water-Resources Investigations Report 84-4143, 15 p.

Ott, R.L., 1993, An introduction to statistical methods and data analyses: Belmont, Calif., Duxbury Press, 1,051 p.

Owenby, J.R., and Ezell, D.S., 1992, Monthly station normals of temperature, precipitation, and heating and cooling degree days 1961-90: Asheville, N.C., National Oceanic and Atmospheric Administration, National Climatic Data Center, Climatography of the United States No. 81, $65 \mathrm{p}$.

Parrett, Charles, Johnson, D.R., and Hull, J.A., 1989, Estimates of monthly streamflow characteristics at selected sites in the upper Missouri River Basin, Montana, base period water years 1937-86: U.S. Geological Survey Water-Resources Investigations Report 89-4082, 103 p.

Parrett, Charles, and Cartier, K.D., 1990, Methods for estimating monthly streamflow characteristics at ungaged sites in western Montana: U.S. Geological Survey Water Supply Paper 2365, 30 p.

Raines, T.H., 1998, Peak-discharge frequency and potential extreme peak discharge for natural streams in the Brazos River Basin, Texas: U.S. Geological Survey WaterResources Investigations Report 98-4178, 42 p.

Riggs, H.C., 1973, Regional analyses of streamflow characteristics: U.S. Geological Survey Techniques of WaterResources Investigations, book 4, chap. B3, 15 p.

Schroeder, E.E., and Massey, B.C., 1977, Technique for estimating the magnitude and frequency of floods in Texas: U.S. Geological Survey Water-Resources Investigations/Open-File Report 77-110, 22 p.

Thomas, D.M., and Benson, M.A., 1970, Generalization of streamflow characteristics from drainage-basin characteristics: U.S. Geological Survey Water-Supply Paper 1975, $55 \mathrm{p}$.

World Meteorological Organization, 1984, Technical regulations: Geneva, Switzerland, v. 1, World Meteorological Organization Publication 49. 


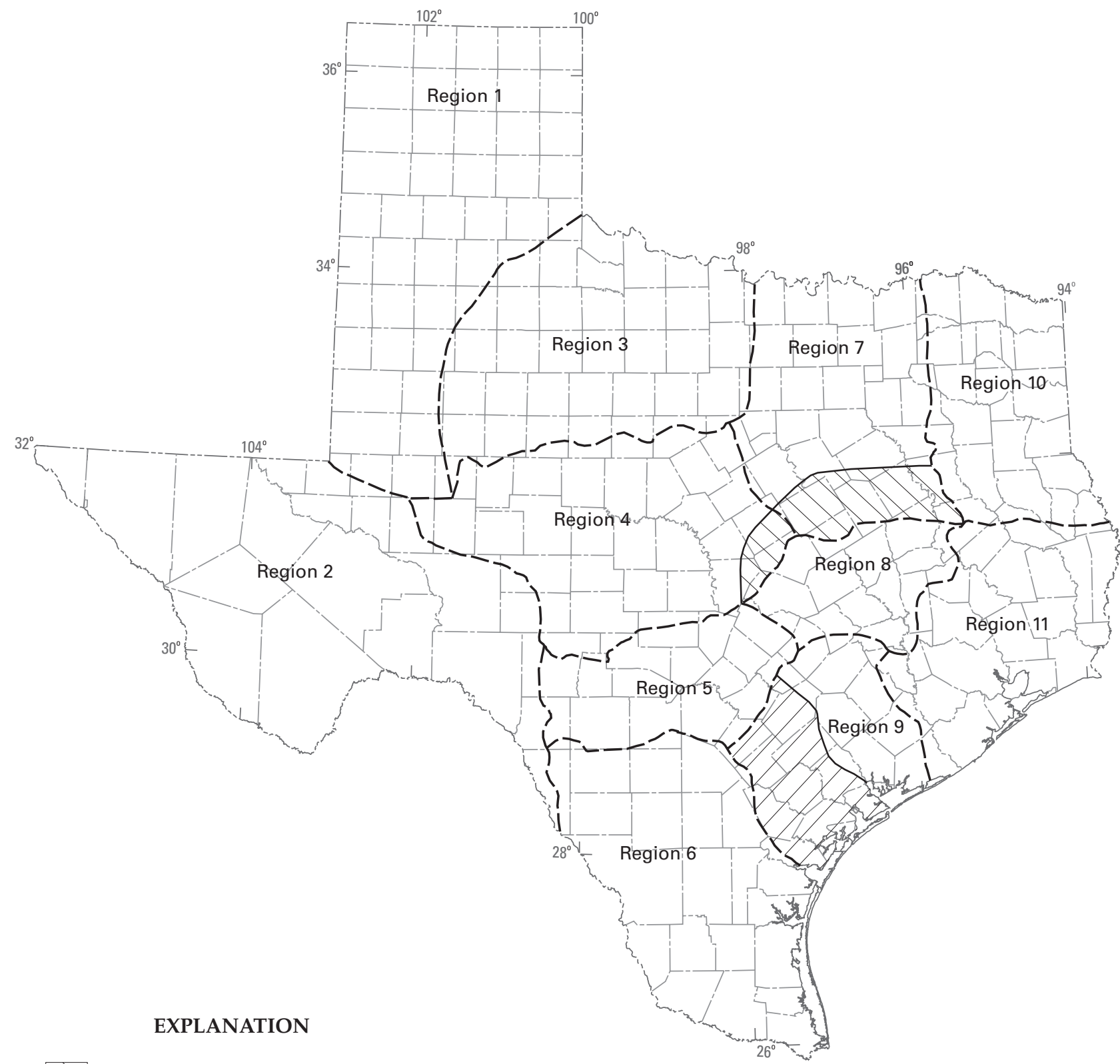

Streamflow-gaging stations also used for region 8 analysis

Streamflow-gaging stations also used for $\begin{array}{lllll}0 & 50 & 100 & 150 & 200 \text { MILES }\end{array}$ region 6 analysis

- - - Hydrologic-region boundary-Modified from Asquith and Slade, 1995

Note: Regions 1 and 2 combined for this study.

Figure 1. Hydrologic regions of Texas (modified from Asquith and Slade, 1995). 


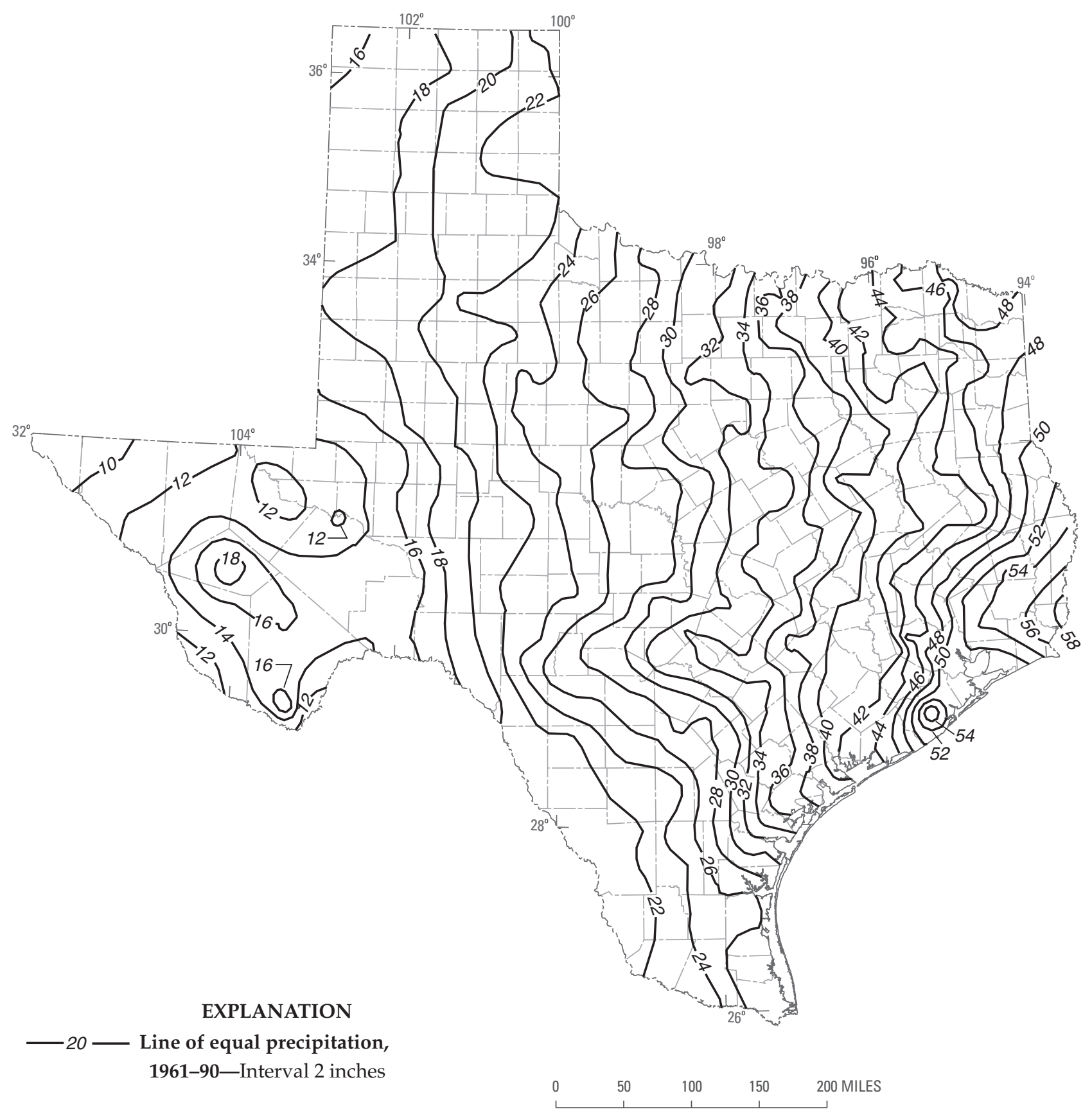

Figure 2. Mean annual precipitation, 1961-90 (data from Owenby and Ezell, 1992). 


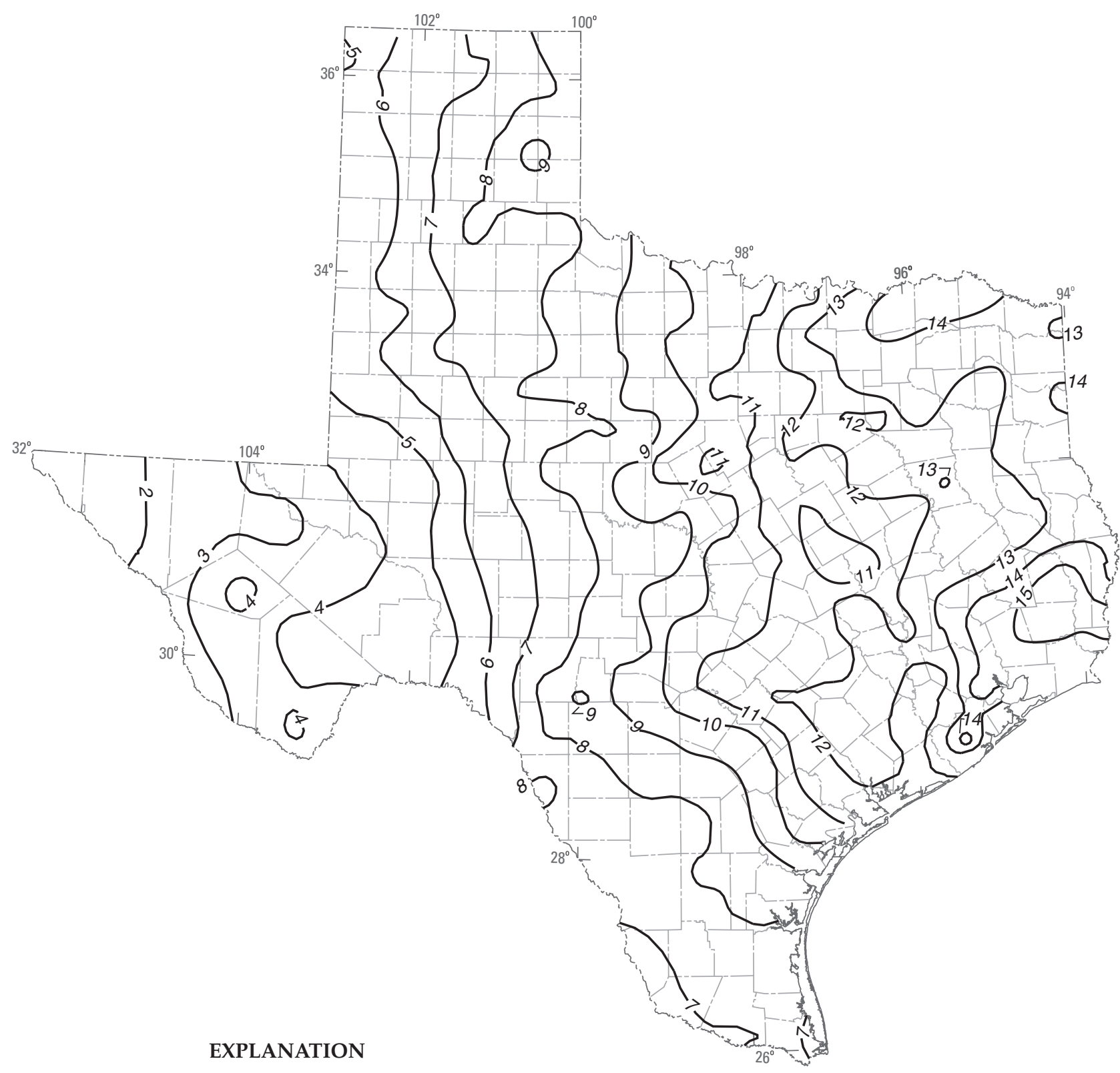

- 10 - Line of equal precipitation, 1961-90-Interval 1 inch

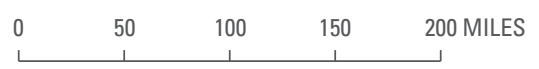

Figure 3. Mean spring precipitation, 1961-90 (data from Owenby and Ezell, 1992). 


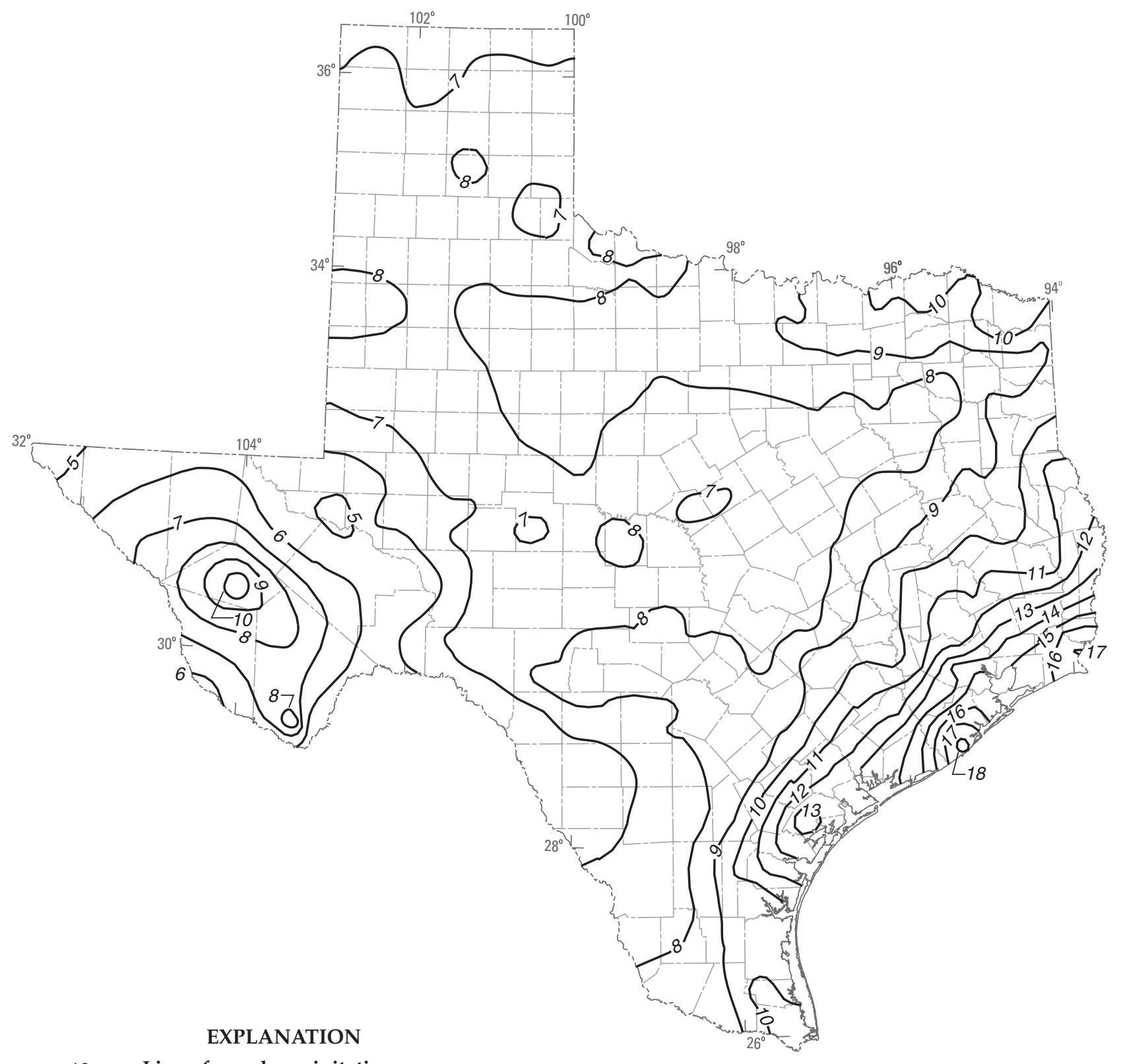

$-10-$ Line of equal precipitation, 1961-90-Interval 1 inch

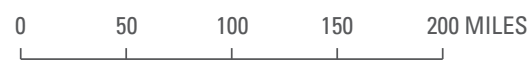

Figure 4. Mean summer precipitation, 1961-90 (data from Owenby and Ezell, 1992). 


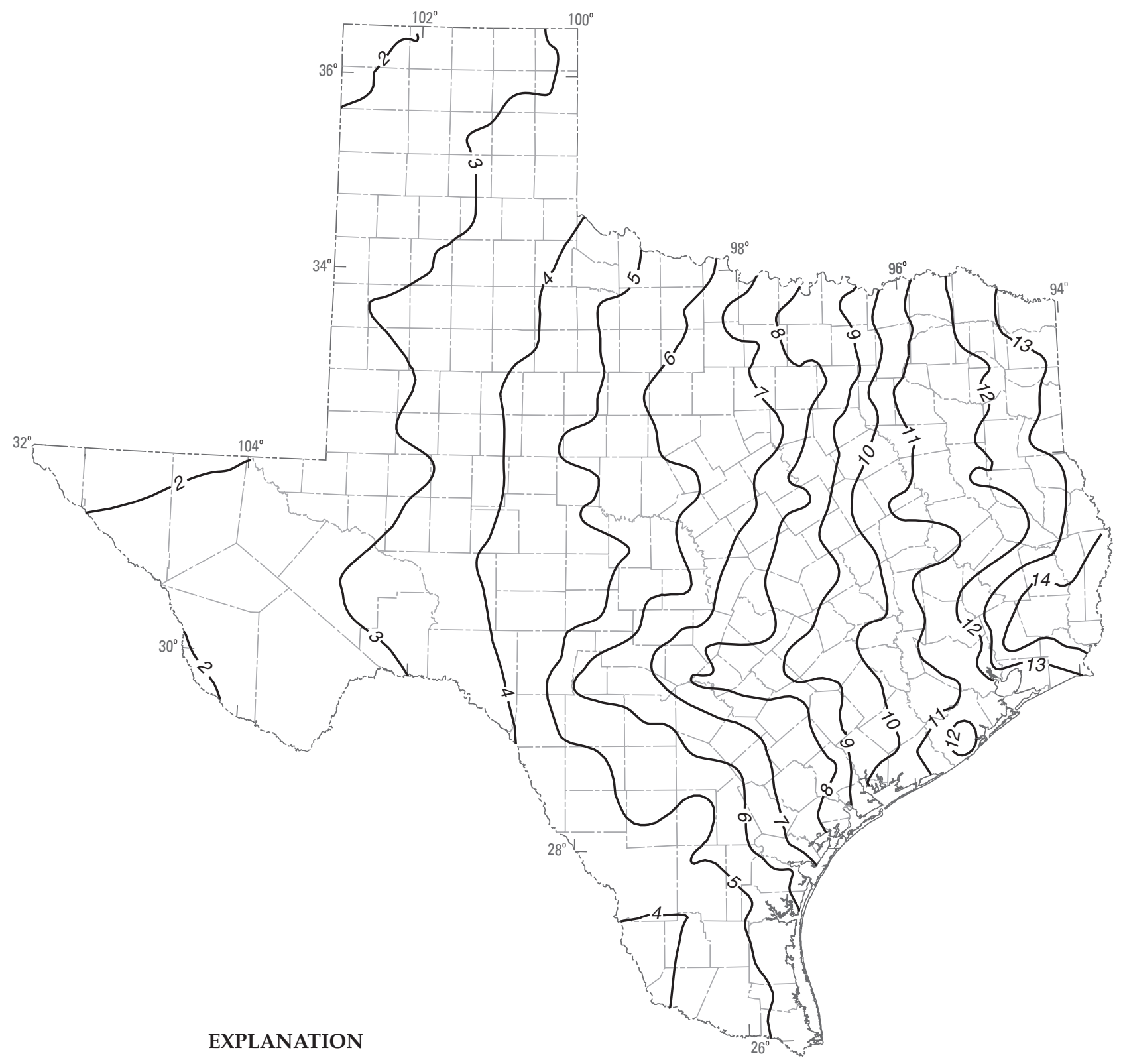

- 10 - Line of equal precipitation, 1961-90-Interval 1 inch

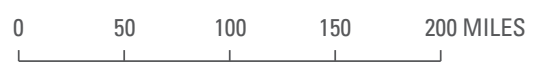

Figure 5. Mean fall precipitation, 1961-90 (data from Owenby and Ezell, 1992). 


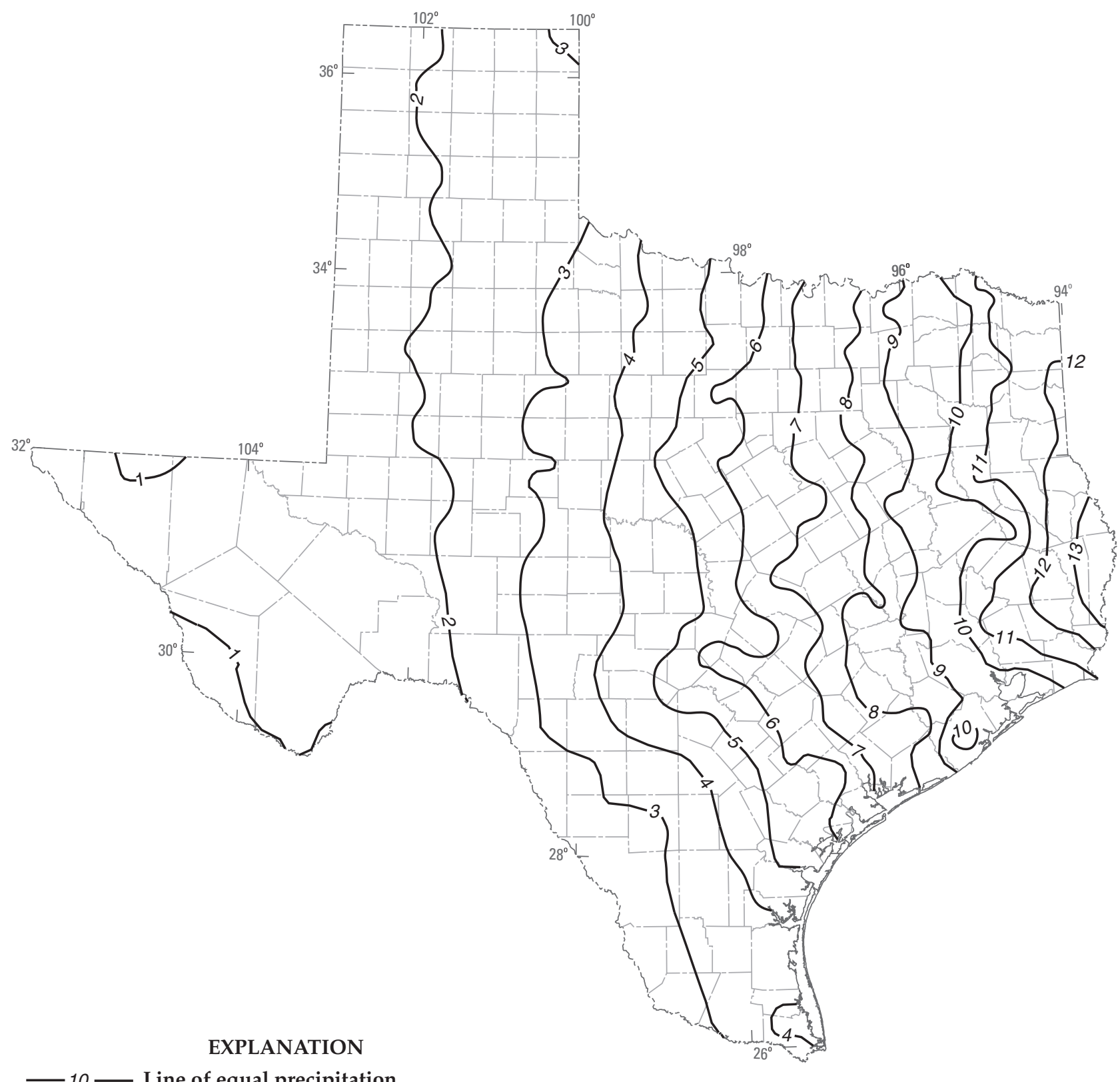

$-10-$ Line of equal precipitation, 1961-90-Interval 1 inch

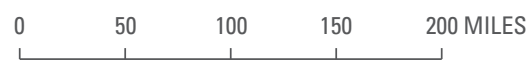

Figure 6. Mean winter precipitation, 1961-90 (data from Owenby and Ezell, 1992). 
Table 1. Basin characteristics for streamflow-gaging stations in or near Texas, with at least 8 years of mean annual $\left[\mathrm{mi}^{2}\right.$, square miles; mi, miles; $\mathrm{ft} / \mathrm{mi}$, feet per mile; in., inches; $\mathrm{ft}{ }^{3} / \mathrm{s}$, cubic feet per second; annual—calendar year; spring-April, and March]

\begin{tabular}{|c|c|c|c|c|c|c|c|c|c|}
\hline $\begin{array}{c}\text { USGS } \\
\text { station } \\
\text { no. }\end{array}$ & $\begin{array}{c}\text { Map } \\
\text { no. } \\
\text { (pl. 1) }\end{array}$ & USGS station name & $\begin{array}{c}\text { Region } \\
\text { no. }\end{array}$ & $\begin{array}{c}\text { Base } \\
\text { station } \\
\text { no. }\end{array}$ & Latitude & Longitude & $\begin{array}{l}\text { First } \\
\text { year } \\
\text { of } \\
\text { record }\end{array}$ & $\begin{array}{l}\text { Last } \\
\text { year } \\
\text { of } \\
\text { record }\end{array}$ & $\begin{array}{l}\text { Weight } \\
\text { factor }\end{array}$ \\
\hline 07154500 & 1 & Cimarron River near Kenton, Oklahoma & 1 & & $36^{\circ} 55^{\prime} 37^{\prime \prime}$ & $102^{\circ} 57^{\prime} 32^{\prime \prime}$ & 1961 & 1990 & 30 \\
\hline 07227920 & 2 & Dixon Creek near Borger, Texas & 1 & 07300500 & $35^{\circ} 39^{\prime} 53^{\prime \prime}$ & $101^{\circ} 21^{\prime} 02^{\prime \prime}$ & 1975 & 1989 & 15 \\
\hline 07232500 & 3 & Beaver River near Guymon, Oklahoma & 1 & & $36^{\circ} 43^{\prime} 23^{\prime \prime}$ & $101^{\circ} 29^{\prime} 31^{\prime \prime}$ & 1961 & 1990 & 30 \\
\hline 07233500 & 4 & Palo Duro Creek near Spearman, Texas & 1 & 07154500 & $36^{\circ} 12^{\prime} 08^{\prime \prime}$ & $101^{\circ} 18^{\prime} 20^{\prime \prime}$ & 1961 & 1979 & 19 \\
\hline 07297910 & 5 & Prairie Dog Town Fork Red River near Wayside, Texas & 1 & 07300500 & $34^{\circ} 50^{\prime} 15^{\prime \prime}$ & $101^{\circ} 24^{\prime} 49^{\prime \prime}$ & 1968 & 1990 & 23 \\
\hline 07298200 & 6 & Tule Creek near Silverton, Texas & 1 & 07232500 & $34^{\circ} 32^{\prime} 36^{\prime \prime}$ & $101^{\circ} 25^{\prime} 46^{\prime \prime}$ & 1965 & 1973 & 9 \\
\hline 07299200 & 7 & Prairie Dog Town Fork Red River near Lakeview, Texas & 1 & 07300500 & $34^{\circ} 34^{\prime} 23^{\prime \prime}$ & $100^{\circ} 44^{\prime} 43^{\prime \prime}$ & 1964 & 1979 & 16 \\
\hline 07299540 & 8 & Prairie Dog Town Fork Red River near Childress, Texas & 1 & 07300500 & $34^{\circ} 34^{\prime} 09^{\prime \prime}$ & $100^{\circ} 11^{\prime} 37^{\prime \prime}$ & 1966 & 1978 & 13 \\
\hline 07299570 & 9 & Red River near Quanah, Texas & 3 & 07300500 & $34^{\circ} 24^{\prime} 47^{\prime \prime}$ & $99^{\circ} 44^{\prime} 03^{\prime \prime}$ & 1961 & 1978 & 18 \\
\hline 07299670 & 10 & Groesbeck Creek at State Highway 6 near Quanah, Texas & 3 & 07308200 & $34^{\circ} 21^{\prime} 16^{\prime \prime}$ & $99^{\circ} 44^{\prime} 24^{\prime \prime}$ & 1963 & 1990 & 28 \\
\hline 07300500 & 11 & Salt Fork Red River at Mangum, Oklahoma & 1 & & $34^{\circ} 51^{\prime} 30^{\prime \prime}$ & $99^{\circ} 30^{\prime} 30^{\prime \prime}$ & 1961 & 1990 & 30 \\
\hline 07301410 & 12 & Sweetwater Creek near Kelton, Texas & 1 & 07300500 & $35^{\circ} 28^{\prime} 23^{\prime \prime}$ & $100^{\circ} 07^{\prime} 14^{\prime \prime}$ & 1963 & 1990 & 28 \\
\hline 07307800 & 13 & Pease River near Childress, Texas & 3 & 07308200 & $34^{\circ} 13 ' 39^{\prime \prime}$ & $100^{\circ} 04^{\prime} 24^{\prime \prime}$ & 1961 & 1990 & 25 \\
\hline 07308200 & 14 & Pease River near Vernon, Texas & 3 & 07307800 & $34^{\circ} 10^{\prime} 44^{\prime \prime}$ & $99^{\circ} 16^{\prime} 40^{\prime \prime}$ & 1961 & 1982 & 22 \\
\hline 07311500 & 15 & Deep Red Run near Randlett, Oklahoma & 3 & & $34^{\circ} 13^{\prime} 16^{\prime \prime}$ & $98^{\circ} 27^{\prime} 11^{\prime \prime}$ & 1961 & 1990 & 30 \\
\hline 07311600 & 16 & North Wichita River near Paducah, Texas & 3 & 07307800 & $33^{\circ} 57^{\prime} 02^{\prime \prime}$ & $100^{\circ} 03^{\prime} 52^{\prime \prime}$ & 1962 & 1982 & 21 \\
\hline 07311700 & 17 & North Wichita River near Truscott, Texas & 3 & & $33^{\circ} 49^{\prime} 14^{\prime \prime}$ & $99^{\circ} 47^{\prime} 10^{\prime \prime}$ & 1961 & 1990 & 30 \\
\hline 07311790 & 18 & South Wichita River at Ross Ranch near Benjamin, Texas & 3 & 07311800 & $33^{\circ} 39^{\prime} 18^{\prime \prime}$ & $100^{\circ} 00^{\prime} 49^{\prime \prime}$ & 1971 & 1979 & 9 \\
\hline 07311800 & 19 & South Wichita River near Benjamin, Texas & 3 & & $33^{\circ} 38^{\prime} 39^{\prime \prime}$ & $99^{\circ} 48^{\prime} 02^{\prime \prime}$ & 1961 & 1990 & 30 \\
\hline 07311900 & 20 & Wichita River near Seymour, Texas & 3 & 07311700 & $33^{\circ} 42^{\prime} 01^{\prime \prime}$ & $99^{\circ} 23^{\prime} 18^{\prime \prime}$ & 1961 & 1979 & 19 \\
\hline 07315200 & 21 & East Fork Little Wichita River near Henrietta, Texas & 3 & 08051000 & $33^{\circ} 48^{\prime} 46^{\prime \prime}$ & $98^{\circ} 05^{\prime} 05^{\prime \prime}$ & 1965 & 1990 & 26 \\
\hline 07315700 & 22 & Mud Creek near Courtney, Oklahoma & 7 & & $34^{\circ} 00^{\prime} 22^{\prime \prime}$ & $97^{\circ} 34^{\prime} 01^{\prime \prime}$ & 1961 & 1990 & 30 \\
\hline 07316200 & 23 & Mineral Creek near Sadler, Texas & 7 & 08049700 & $33^{\circ} 42^{\prime} 08^{\prime \prime}$ & $96^{\circ} 50^{\prime} 51^{\prime \prime}$ & 1969 & 1976 & 8 \\
\hline 07332500 & 24 & Blue River near Blue, Oklahoma & 7 & & $34^{\circ} 00^{\prime} 00^{\prime \prime}$ & $96^{\circ} 14^{\prime} 24^{\prime \prime}$ & 1961 & 1990 & 30 \\
\hline 07332600 & 25 & Bois d'Arc Creek near Randolph, Texas & 7 & 07343000 & $33^{\circ} 28^{\prime} 32^{\prime \prime}$ & $96^{\circ} 12^{\prime} 52^{\prime \prime}$ & 1964 & 1985 & 22 \\
\hline 07336750 & 26 & Little Pine Creek near Kanawha, Texas & 10 & 07343000 & $33^{\circ} 50^{\prime} 26^{\prime \prime}$ & $95^{\circ} 15^{\prime} 55^{\prime \prime}$ & 1970 & 1980 & 11 \\
\hline 07336800 & 27 & Pecan Bayou near Clarksville, Texas & 10 & 07342500 & $33^{\circ} 41^{\prime} 07^{\prime \prime}$ & $94^{\circ} 59^{\prime} 41^{\prime \prime}$ & 1963 & 1977 & 15 \\
\hline 07342470 & 28 & South Sulphur River near Commerce, Texas & 7 & 07342500 & $33^{\circ} 13^{\prime} 11^{\prime \prime}$ & $95^{\circ} 51^{\prime} 45^{\prime}$ & 1980 & 1990 & 11 \\
\hline 07342500 & 29 & South Sulphur River near Cooper, Texas & 10 & & $33^{\circ} 21^{\prime} 20^{\prime \prime}$ & $95^{\circ} 35^{\prime} 39^{\prime \prime}$ & 1961 & 1990 & 30 \\
\hline 07343000 & 30 & North Sulphur River near Cooper, Texas & 10 & & $33^{\circ} 28^{\prime} 29^{\prime \prime}$ & $95^{\circ} 35^{\prime} 15^{\prime \prime}$ & 1961 & 1990 & 30 \\
\hline 07343200 & 31 & Sulphur River near Talco, Texas & 10 & & $33^{\circ} 23^{\prime} 10^{\prime \prime}$ & $95^{\circ} 07 ' 56^{\prime \prime}$ & 1961 & 1990 & 30 \\
\hline 07343300 & 32 & Cuthand Creek near Bogata, Texas & 10 & 07342500 & $33^{\circ} 32^{\prime} 51^{\prime \prime}$ & $95^{\circ} 10^{\prime} 22^{\prime \prime}$ & 1964 & 1974 & 11 \\
\hline 07343500 & 33 & White Oak Creek near Talco, Texas & 10 & 07342500 & $33^{\circ} 19^{\prime} 20^{\prime \prime}$ & $95^{\circ} 05^{\prime} 33^{\prime \prime}$ & 1961 & 1972 & 12 \\
\hline 07344486 & 34 & Brushy Creek at Scroggins, Texas & 10 & 08017200 & $32^{\circ} 58^{\prime} 32^{\prime \prime}$ & $95^{\circ} 11^{\prime} 03^{\prime \prime}$ & 1979 & 1990 & 12 \\
\hline 07345000 & 35 & Boggy Creek near Daingerfield, Texas & 10 & 07346140 & $33^{\circ} 02^{\prime} 10^{\prime \prime}$ & $94^{\circ} 47^{\prime} 15^{\prime \prime}$ & 1961 & 1977 & 17 \\
\hline
\end{tabular}

Footnote at end of table. 
streamflow data, 1961-90

May, and June; summer—July, August, and September; fall—October, November, and December; winter—January, February,

\begin{tabular}{|c|c|c|c|c|c|c|c|c|c|c|c|c|c|c|}
\hline \multirow{2}{*}{$\begin{array}{c}\text { Map } \\
\text { no. } \\
\text { (pl. 1) }\end{array}$} & \multirow{2}{*}{$\begin{array}{l}\text { Contri- } \\
\text { buting } \\
\text { drainage } \\
\text { area } \\
(\mathrm{A}) \\
\left(\mathrm{mi}^{2}\right)\end{array}$} & \multirow{2}{*}{$\begin{array}{l}\text { Main } \\
\text { stream } \\
\text { length } \\
(\mathrm{L}) \\
(\mathrm{mi})\end{array}$} & \multirow{2}{*}{$\begin{array}{c}\text { Stream } \\
\text { slope } \\
(\mathrm{SL}) \\
(\mathrm{ft} / \mathrm{mi})\end{array}$} & \multirow{2}{*}{$\begin{array}{c}\text { Basin } \\
\text { shape } \\
\text { factor } \\
\text { (SH) }\end{array}$} & \multicolumn{5}{|c|}{$\begin{array}{l}\text { Precipitation } \\
\text { (in.) }\end{array}$} & \multicolumn{5}{|c|}{$\begin{array}{l}\text { Estimated 1961-90 streamflow } \\
\qquad\left(\mathrm{ft}^{3} / \mathrm{s}\right)\end{array}$} \\
\hline & & & & & Annual & Spring & $\begin{array}{l}\text { Sum- } \\
\text { mer }\end{array}$ & Fall & $\begin{array}{l}\text { Win- } \\
\text { ter }\end{array}$ & Annual & Spring & $\begin{array}{l}\text { Sum- } \\
\text { mer }\end{array}$ & Fall & $\begin{array}{l}\text { Win- } \\
\text { ter }\end{array}$ \\
\hline 1 & 1,038 & 104 & 26.2 & 10.42 & 16.38 & 5.59 & 7.19 & 1.97 & 1.63 & 18.20 & 26.05 & 39.06 & 5.77 & 1.91 \\
\hline 2 & 134 & 27.3 & 23.55 & 5.56 & 20.6 & 7.53 & 7.56 & 2.91 & 2.6 & 1.98 & 4.49 & 2.58 & 0.23 & 0.61 \\
\hline 3 & 1,175 & 203 & 14.8 & 35.07 & 16.65 & 6.12 & 7.07 & 1.9 & 1.56 & 10.50 & 18.99 & 11.66 & 6.14 & 5.21 \\
\hline 4 & 440 & 78.43 & 10.17 & 13.98 & 17.48 & 6.48 & 6.74 & 2.21 & 2.05 & 12.05 & 25.32 & 14.31 & 7.68 & 0.90 \\
\hline 5 & 930 & 211.12 & 11.24 & 47.93 & 17.67 & 6.02 & 7.36 & 2.51 & 1.78 & 35.15 & 42.07 & 70.26 & 24.49 & 3.80 \\
\hline 6 & 190 & 29.97 & 6.84 & 4.73 & 19 & 6.89 & 7.33 & 2.8 & 1.98 & 6.77 & 22.36 & 4.20 & 0.32 & 0.21 \\
\hline 7 & 2,023 & 267.34 & 10.87 & 35.33 & 18.8 & 6.68 & 7.5 & 2.68 & 1.94 & 65.69 & 150.43 & 84.90 & 15.72 & 11.72 \\
\hline 8 & 2,956 & 308.17 & 10.38 & 32.13 & 18.89 & 6.76 & 7.41 & 2.73 & 1.99 & 88.33 & 195.11 & 110.09 & 25.68 & 22.42 \\
\hline 9 & 3,552 & 346.44 & 9.87 & 33.79 & 19.11 & 6.86 & 7.4 & 2.81 & 2.04 & 113.42 & 236.57 & 125.72 & 53.86 & 37.51 \\
\hline 10 & 303 & 42.06 & 10.22 & 5.84 & 22.87 & 7.95 & 7.86 & 4.09 & 2.97 & 15.58 & 19.77 & 20.48 & 16.06 & 6.00 \\
\hline 11 & 1,357 & 132 & 13.8 & 12.84 & 22.18 & 8.57 & 7.44 & 3.48 & 2.69 & 78.63 & 159.98 & 46.81 & 56.11 & 51.61 \\
\hline 12 & 267 & 45.23 & 15.89 & 7.66 & 22.05 & 8.65 & 7.4 & 3.21 & 2.79 & 14.77 & 22.59 & 7.02 & 13.68 & 15.78 \\
\hline 13 & 2,195 & 108.4 & 16.22 & 5.35 & 21.15 & 7.63 & 7.53 & 3.51 & 2.48 & 56.71 & 97.54 & 60.67 & 50.69 & 17.96 \\
\hline 14 & 2,929 & 179.28 & 11.6 & 10.97 & 21.82 & 7.78 & 7.58 & 3.75 & 2.71 & 111.08 & 197.00 & 125.84 & 88.10 & 33.36 \\
\hline 15 & 617 & 64.2 & 8.51 & 6.68 & 28.61 & 10.44 & 8.31 & 5.46 & 4.4 & 162.44 & 291.07 & 105.75 & 154.26 & 98.67 \\
\hline 16 & 540 & 61.14 & 14.49 & 6.92 & 22.84 & 8.11 & 7.71 & 4.07 & 2.95 & 22.60 & 42.66 & 21.91 & 16.50 & 9.33 \\
\hline 17 & 937 & 95.9 & 11.12 & 9.82 & 23.43 & 8.26 & 7.72 & 4.33 & 3.12 & 62.98 & 91.97 & 76.69 & 57.86 & 25.39 \\
\hline 18 & 499 & 75.6 & 11.76 & 11.45 & 23.75 & 8.23 & 8.23 & 4.18 & 3.11 & 13.35 & 20.32 & 13.46 & 13.60 & 6.01 \\
\hline 19 & 584 & 103.2 & 9.63 & 18.24 & 24.01 & 8.29 & 8.2 & 4.33 & 3.19 & 40.67 & 62.30 & 47.25 & 38.91 & 14.20 \\
\hline 20 & 1,874 & 155.18 & 7.57 & 12.85 & 24.02 & 8.38 & 7.91 & 4.46 & 3.27 & 151.94 & 219.00 & 181.38 & 137.82 & 69.55 \\
\hline 21 & 178 & 31.32 & 8.9 & 5.51 & 31.75 & 10.87 & 8.31 & 7.05 & 5.52 & 30.32 & 63.54 & 11.61 & 22.51 & 23.62 \\
\hline 22 & 572 & 45 & 6.5 & 3.54 & 33.57 & 11.96 & 8.88 & 7.05 & 5.68 & 177.36 & 390.67 & 56.18 & 106.30 & 156.27 \\
\hline 23 & 26 & 11.09 & 16.57 & 4.73 & 37.1 & 11.98 & 9.23 & 8.45 & 7.44 & 7.77 & 11.90 & 2.09 & 3.91 & 13.18 \\
\hline 24 & 476 & 112 & 5.99 & 26.35 & 40.53 & 13.8 & 9.76 & 9.27 & 7.7 & 316.17 & 516.03 & 110.08 & 284.33 & 354.22 \\
\hline 25 & 72 & 17.97 & 10.41 & 4.49 & 42.93 & 14.17 & 9.77 & 10.05 & 8.94 & 59.29 & 79.94 & 25.03 & 67.48 & 64.72 \\
\hline 26 & 75.4 & 19.6 & 6.53 & 5.1 & 46.89 & 14.45 & 10.02 & 11.96 & 10.46 & 64.23 & 66.60 & 10.62 & 85.55 & 94.17 \\
\hline 27 & 100 & 27.52 & 5.48 & 7.57 & 47.28 & 14.53 & 9.66 & 12.34 & 10.75 & 76.91 & 99.64 & 6.00 & 83.48 & 118.52 \\
\hline 28 & 189 & 33.95 & 6.57 & 6.1 & 42.97 & 13.74 & 9.56 & 10.59 & 9.08 & 171.54 & 259.52 & 57.97 & 141.44 & 227.20 \\
\hline 29 & 527 & 64.94 & 4.75 & 8 & 43.47 & 13.7 & 9.57 & 10.89 & 9.31 & 439.69 & 651.21 & 114.88 & 441.98 & 550.69 \\
\hline 30 & 276 & 41.32 & 6.83 & 6.18 & 43.98 & 13.88 & 9.84 & 10.97 & 9.29 & 252.39 & 361.65 & 81.54 & 261.41 & 304.96 \\
\hline 31 & 1,365 & 109.28 & 3.54 & 8.75 & 43.95 & 13.55 & 9.76 & 11.23 & 9.41 & 1395.50 & 2054.69 & 348.08 & 1433.69 & 1745.56 \\
\hline 32 & 69 & 20.47 & 7.85 & 6.07 & 44.9 & 13.39 & 9.84 & 12.08 & 9.59 & 64.28 & 85.93 & 8.66 & 67.09 & 95.47 \\
\hline 33 & 494 & 69.05 & 3.94 & 9.65 & 44.38 & 13.5 & 9.19 & 11.98 & 9.71 & 399.35 & 641.40 & 55.23 & 408.47 & 492.31 \\
\hline 34 & 23.4 & 6.43 & 24.49 & 1.77 & 42.9 & 13.12 & 8.25 & 11.4 & 10.13 & 10.16 & 11.88 & 3.77 & 12.50 & 12.49 \\
\hline 35 & 72 & 17.48 & 6.94 & 4.25 & 46.97 & 13.62 & 9.52 & 12.79 & 11.04 & 69.46 & 83.98 & 12.90 & 54.24 & 126.73 \\
\hline
\end{tabular}


Table 1. Basin characteristics for streamflow-gaging stations in or near Texas, with at least 8 years of mean annual

\begin{tabular}{|c|c|c|c|c|c|c|c|c|c|}
\hline $\begin{array}{c}\text { USGS } \\
\text { station } \\
\text { no. }\end{array}$ & $\begin{array}{c}\text { Map } \\
\text { no. } \\
\text { (pl. 1) }\end{array}$ & USGS station name & $\begin{array}{c}\text { Region } \\
\text { no. }\end{array}$ & $\begin{array}{c}\text { Base } \\
\text { station } \\
\text { no. }\end{array}$ & Latitude & Longitude & $\begin{array}{l}\text { First } \\
\text { year } \\
\text { of } \\
\text { record }\end{array}$ & $\begin{array}{l}\text { Last } \\
\text { year } \\
\text { of } \\
\text { record }\end{array}$ & $\begin{array}{l}\text { Weight } \\
\text { factor }\end{array}$ \\
\hline 07346045 & 36 & Black Cypress Bayou at Jefferson, Texas & 10 & 07346070 & $32^{\circ} 46^{\prime} 40^{\prime \prime}$ & $94^{\circ} 21^{\prime} 26^{\prime \prime}$ & 1969 & 1990 & 22 \\
\hline 07346050 & 37 & Little Cypress Creek near Ore City, Texas & 10 & 07346070 & $32^{\circ} 40^{\prime} 21^{\prime \prime}$ & $94^{\circ} 45^{\prime} 03^{\prime \prime}$ & 1964 & 1990 & 27 \\
\hline 07346070 & 38 & Little Cypress Creek near Jefferson, Texas & 10 & & $32^{\circ} 42^{\prime} 50^{\prime \prime}$ & $94^{\circ} 20^{\prime} 44^{\prime \prime}$ & 1961 & 1990 & 30 \\
\hline 07346140 & 39 & Frazier Creek near Linden, Texas & 10 & 07345000 & $33^{\circ} 03^{\prime} 14^{\prime \prime}$ & $94^{\circ} 17^{\prime} 24^{\prime \prime}$ & 1966 & 1990 & 25 \\
\hline 08017200 & 40 & Cowleech Fork Sabine River at Greenville, Texas & 7 & & $33^{\circ} 07^{\prime} 58^{\prime \prime}$ & $96^{\circ} 04^{\prime} 36^{\prime \prime}$ & 1961 & 1990 & 30 \\
\hline 08017300 & 41 & South Fork Sabine River near Quilan, Texas & 7 & & $32^{\circ} 53^{\prime} 52^{\prime \prime}$ & $96^{\circ} 15^{\prime} 11^{\prime \prime}$ & 1961 & 1990 & 30 \\
\hline 08018730 & 42 & Burke Creek near Yantis, Texas & 10 & 08049700 & $32^{\circ} 59^{\prime} 26^{\prime \prime}$ & $95^{\circ} 37^{\prime} 18^{\prime \prime}$ & 1979 & 1989 & 11 \\
\hline 08019000 & 43 & Lake Fork Creek near Quitman, Texas & 10 & 07343000 & $32^{\circ} 45^{\prime} 47^{\prime \prime}$ & $95^{\circ} 27^{\prime} 46^{\prime \prime}$ & 1961 & 1979 & 19 \\
\hline 08020200 & 44 & Prairie Creek near Gladewater, Texas & 10 & 08070500 & $32^{\circ} 28^{\prime} 45^{\prime \prime}$ & $94^{\circ} 57^{\prime} 14^{\prime \prime}$ & 1969 & 1976 & 8 \\
\hline 08020700 & 45 & Rabbit Creek at Kilgore, Texas & 10 & 07346070 & $32^{\circ} 23^{\prime} 17^{\prime \prime}$ & $94^{\circ} 54^{\prime} 11^{\prime \prime}$ & 1964 & 1976 & 13 \\
\hline 08022400 & 46 & Socagee Creek near Carthage, Texas & 10 & 07346070 & $32^{\circ} 13^{\prime} 54^{\prime \prime}$ & $94^{\circ} 05^{\prime} 31^{\prime \prime}$ & 1963 & 1973 & 11 \\
\hline 08023200 & 47 & Tenaha Creek near Shelbyville, Texas & 10 & 08066170 & $31^{\circ} 45^{\prime} 56^{\prime \prime}$ & $94^{\circ} 05^{\prime} 02^{\prime \prime}$ & 1961 & 1980 & 20 \\
\hline 08029500 & 48 & Big Cow Creek near Newton, Texas & 11 & & $30^{\circ} 49^{\prime} 08^{\prime \prime}$ & $93^{\circ} 47^{\prime} 07^{\prime \prime}$ & 1961 & 1990 & 30 \\
\hline 08030000 & 49 & Cypress Creek near Buna, Texas & 11 & 08066170 & $30^{\circ} 25^{\prime} 52^{\prime \prime}$ & $93^{\circ} 54^{\prime} 28^{\prime \prime}$ & 1961 & 1983 & 23 \\
\hline 08031000 & 50 & Cow Bayou near Mauriceville, Texas & 11 & 08041500 & $30^{\circ} 11^{\prime} 10^{\prime \prime}$ & $93^{\circ} 54^{\prime} 30^{\prime \prime}$ & 1961 & 1986 & 26 \\
\hline 08031200 & 51 & Kickapoo Creek near Brownsboro, Texas & 10 & 07342500 & $32^{\circ} 18^{\prime} 34^{\prime \prime}$ & $95^{\circ} 36^{\prime} 19^{\prime \prime}$ & 1963 & 1989 & 27 \\
\hline 08033300 & 52 & Piney Creek near Groveton, Texas & 11 & 08068500 & $31^{\circ} 08^{\prime} 25^{\prime \prime}$ & $95^{\circ} 05^{\prime} 11^{\prime \prime}$ & 1962 & 1989 & 28 \\
\hline 08033900 & 53 & East Fork Angelina River near Cushing, Texas & 10 & 08041500 & $31^{\circ} 51^{\prime} 36^{\prime \prime}$ & $94^{\circ} 49^{\prime} 23^{\prime \prime}$ & 1965 & 1989 & 25 \\
\hline 08037050 & 54 & Bayou LaNana at Nacogdoches, Texas & 10 & 08070000 & $31^{\circ} 36^{\prime} 58^{\prime \prime}$ & $94^{\circ} 38^{\prime} 28^{\prime \prime}$ & 1965 & 1990 & 24 \\
\hline 08038000 & 55 & Attoyac Bayou near Chireno, Texas & 10 & 08070000 & $31^{\circ} 30^{\prime} 15^{\prime \prime}$ & $94^{\circ} 18^{\prime} 15^{\prime \prime}$ & 1961 & 1989 & 26 \\
\hline 08039100 & 56 & Ayish Bayou near San Augustine, Texas & 10 & 08066170 & $31^{\circ} 23^{\prime} 46^{\prime \prime}$ & $94^{\circ} 09^{\prime} 03^{\prime \prime}$ & 1961 & 1985 & 25 \\
\hline 08039500 & 57 & Angelina River at Horger, Texas & 11 & 07343200 & $31^{\circ} 02^{\prime} 08^{\prime \prime}$ & $94^{\circ} 07^{\prime} 48^{\prime \prime}$ & 1966 & 1973 & 8 \\
\hline 08041500 & 58 & Village Creek near Kountze, Texas & 11 & & $30^{\circ} 23^{\prime} 52^{\prime \prime}$ & $94^{\circ} 15^{\prime} 48^{\prime \prime}$ & 1961 & 1990 & 30 \\
\hline 08041700 & 59 & Pine Island Bayou near Sour Lake, Texas & 11 & 08071000 & $30^{\circ} 06^{\prime} 21^{\prime \prime}$ & $94^{\circ} 20^{\prime} 04^{\prime \prime}$ & 1968 & 1990 & 23 \\
\hline 08042800 & 60 & West Fork Trinity River near Jacksboro, Texas & 3 & 07311700 & $33^{\circ} 17^{\prime} 36^{\prime \prime}$ & $98^{\circ} 04^{\prime} 43^{\prime \prime}$ & 1961 & 1973 & 13 \\
\hline 08049550 & 61 & Big Bear Creek near Grapevine, Texas & 7 & 08049700 & $32^{\circ} 54^{\prime} 48^{\prime \prime}$ & $97^{\circ} 07^{\prime} 44^{\prime \prime}$ & 1968 & 1979 & 12 \\
\hline 08049700 & 62 & Walnut Creek near Mansfield, Texas & 7 & & $32^{\circ} 34^{\prime} 51^{\prime \prime}$ & $97^{\circ} 06^{\prime} 06^{\prime \prime}$ & 1961 & 1990 & 30 \\
\hline 08051000 & 63 & Isle Du Bois Creek near Pilot Point, Texas & 7 & 07315200 & $33^{\circ} 24^{\prime} 23^{\prime \prime}$ & $97^{\circ} 00^{\prime} 45^{\prime \prime}$ & 1961 & 1984 & 24 \\
\hline 08051500 & 64 & Clear Creek near Sanger, Texas & 7 & 07342500 & $33^{\circ} 20^{\prime} 21^{\prime \prime}$ & $97^{\circ} 10^{\prime} 51^{\prime \prime}$ & 1961 & 1980 & 20 \\
\hline 08061540 & 65 & Rowlett Creek near Sachse, Texas & 7 & 08051000 & $32^{\circ} 57^{\prime} 35^{\prime \prime}$ & $96^{\circ} 36^{\prime} 51^{\prime \prime}$ & 1969 & 1990 & 22 \\
\hline 08062900 & 66 & Kings Creek near Kaufman, Texas & 7 & 07343000 & $32^{\circ} 30^{\prime} 48^{\prime \prime}$ & $96^{\circ} 19^{\prime} 44^{\prime \prime}$ & 1964 & 1971 & 8 \\
\hline 08064700 & 67 & Tehuacana Creek near Streetman, Texas & $7^{1}(8)$ & 08095400 & $31^{\circ} 50^{\prime} 54^{\prime \prime}$ & $96^{\circ} 17^{\prime} 23^{\prime \prime}$ & 1969 & 1990 & 22 \\
\hline 08064800 & 68 & Catfish Creek near Tennessee Colony, Texas & $7^{1}(8)$ & 08049700 & $31^{\circ} 52^{\prime} 51^{\prime \prime}$ & $95^{\circ} 52^{\prime} 07^{\prime \prime}$ & 1963 & 1989 & 27 \\
\hline 08065200 & 69 & Upper Keechi Creek near Oakwood, Texas & $7^{1}(8)$ & 08095300 & $31^{\circ} 34^{\prime} 11^{\prime \prime}$ & $95^{\circ} 53^{\prime} 17^{\prime \prime}$ & 1963 & 1990 & 28 \\
\hline 08065700 & 70 & Caney Creek near Madisonville, Texas & 8 & 07343000 & $30^{\circ} 56^{\prime} 12^{\prime \prime}$ & $95^{\circ} 56^{\prime} 07^{\prime \prime}$ & 1964 & 1976 & 13 \\
\hline
\end{tabular}

Footnote at end of table. 
streamflow data, 1961-90-Continued

\begin{tabular}{|c|c|c|c|c|c|c|c|c|c|c|c|c|c|c|}
\hline \multirow{2}{*}{$\begin{array}{c}\text { Map } \\
\text { no. } \\
\text { (pl. 1) }\end{array}$} & \multirow{2}{*}{$\begin{array}{l}\text { Contri- } \\
\text { buting } \\
\text { drainage } \\
\text { area } \\
(\mathrm{A}) \\
\left(\mathrm{mi}^{2}\right)\end{array}$} & \multirow{2}{*}{$\begin{array}{c}\text { Main } \\
\text { stream } \\
\text { length } \\
(\mathrm{L}) \\
(\mathrm{mi})\end{array}$} & \multirow{2}{*}{$\begin{array}{c}\text { Stream } \\
\text { slope } \\
(\mathrm{SL}) \\
(\mathrm{ft} / \mathrm{mi})\end{array}$} & \multirow{2}{*}{$\begin{array}{c}\text { Basin } \\
\text { shape } \\
\text { factor } \\
\text { (SH) }\end{array}$} & \multicolumn{5}{|c|}{$\begin{array}{l}\text { Precipitation } \\
\text { (in.) }\end{array}$} & \multicolumn{5}{|c|}{$\begin{array}{l}\text { Estimated } 1961-90 \text { streamflow } \\
\left(\mathrm{ft}^{3} / \mathrm{s}\right)\end{array}$} \\
\hline & & & & & Annual & Spring & $\begin{array}{l}\text { Sum- } \\
\text { mer }\end{array}$ & Fall & $\begin{array}{c}\text { Win- } \\
\text { ter }\end{array}$ & Annual & Spring & $\begin{array}{l}\text { Sum- } \\
\text { mer }\end{array}$ & Fall & $\begin{array}{l}\text { Win- } \\
\text { ter }\end{array}$ \\
\hline 36 & 365 & 53.37 & 3.75 & 7.8 & 46.6 & 13.55 & 8.98 & 12.73 & 11.34 & 308.40 & 439.55 & 47.66 & 236.43 & 509.95 \\
\hline 37 & 383 & 47.76 & 4.47 & 5.96 & 44.44 & 12.95 & 8.66 & 12.01 & 10.82 & 276.68 & 416.53 & 48.64 & 194.09 & 447.45 \\
\hline 38 & 675 & 85.52 & 3.14 & 10.84 & 45.31 & 13.13 & 8.75 & 12.31 & 11.12 & 488.42 & 751.43 & 82.30 & 322.47 & 797.50 \\
\hline 39 & 48 & 11.22 & 10.53 & 2.62 & 48.37 & 14.11 & 9.33 & 13.07 & 11.86 & 40.23 & 51.16 & 5.59 & 33.24 & 70.94 \\
\hline 40 & 77.7 & 19.03 & 8.96 & 4.66 & 42 & 13.41 & 9.41 & 10.17 & 9.01 & 63.28 & 99.27 & 21.65 & 55.49 & 76.69 \\
\hline 41 & 78.7 & 18.48 & 8.88 & 4.34 & 40.27 & 13.27 & 8.65 & 9.67 & 8.68 & 80.89 & 131.77 & 22.87 & 72.08 & 96.85 \\
\hline 42 & 33.1 & 9.77 & 10.41 & 2.88 & 45.33 & 14.17 & 9.38 & 11.92 & 9.86 & 19.04 & 30.70 & 3.93 & 12.09 & 29.43 \\
\hline 43 & 585 & 50.05 & 4.92 & 4.28 & 43.37 & 13.66 & 8.6 & 11.34 & 9.77 & 413.88 & 635.68 & 51.24 & 385.19 & 583.41 \\
\hline 44 & 48.9 & 14.11 & 14.88 & 4.07 & 43.97 & 12.94 & 8.5 & 11.65 & 10.88 & 22.44 & 26.24 & 8.62 & 16.58 & 38.33 \\
\hline 45 & 75.8 & 13.91 & 13.91 & 2.55 & 45.04 & 12.94 & 8.75 & 12.26 & 11.09 & 47.96 & 68.28 & 12.53 & 32.90 & 78.14 \\
\hline 46 & 82.6 & 18.17 & 6.86 & 4 & 50.66 & 14.22 & 10.12 & 13.88 & 12.44 & 62.74 & 53.95 & 24.95 & 52.75 & 119.31 \\
\hline 47 & 97.8 & 19.69 & 9 & 3.96 & 49.93 & 13.67 & 11.23 & 12.7 & 12.33 & 69.04 & 76.51 & 18.88 & 54.16 & 126.63 \\
\hline 48 & 128 & 25.84 & 12.06 & 5.22 & 53.51 & 14.21 & 11.97 & 13.92 & 13.41 & 126.56 & 134.66 & 64.41 & 118.99 & 188.18 \\
\hline 49 & 69.2 & 18.24 & 4.5 & 4.81 & 54.97 & 14.89 & 12.9 & 14.08 & 13.1 & 77.98 & 73.64 & 21.04 & 74.23 & 143.02 \\
\hline 50 & 83.3 & 25.2 & 2.6 & 7.62 & 56.64 & 15.08 & 14.45 & 14.26 & 12.85 & 100.96 & 111.95 & 43.64 & 79.76 & 168.48 \\
\hline 51 & 232 & 35.52 & 4.34 & 5.44 & 41.4 & 12.91 & 7.7 & 11.26 & 9.53 & 137.93 & 230.06 & 15.95 & 101.87 & 203.82 \\
\hline 52 & 79 & 21.2 & 7.58 & 5.69 & 43.76 & 12.33 & 10.06 & 11.35 & 10.02 & 42.02 & 56.81 & 12.12 & 30.15 & 69.00 \\
\hline 53 & 158 & 27.23 & 7.47 & 4.69 & 45.25 & 13.01 & 8.84 & 12.03 & 11.37 & 110.82 & 138.68 & 39.83 & 91.19 & 173.59 \\
\hline 54 & 31.3 & 13.16 & 13.46 & 5.54 & 45.05 & 12.85 & 9.2 & 11.86 & 11.14 & 30.24 & 41.31 & 6.18 & 24.51 & 48.95 \\
\hline 55 & 503 & 76.14 & 4.44 & 11.52 & 47.57 & 13.24 & 10.27 & 12.3 & 11.76 & 406.99 & 518.47 & 128.56 & 340.20 & 640.73 \\
\hline 56 & 89 & 22.18 & 10.06 & 5.53 & 50.34 & 13.6 & 11.37 & 12.76 & 12.61 & 86.83 & 98.00 & 18.43 & 71.20 & 159.68 \\
\hline 57 & 3,486 & 184.31 & 2.35 & 9.74 & 46.24 & 13.03 & 9.62 & 12.17 & 11.42 & 2208.62 & 3099.38 & 875.44 & 1205.67 & 3654.00 \\
\hline 58 & 860 & 78.56 & 4.47 & 7.18 & 52.83 & 15.4 & 11.33 & 13.97 & 12.13 & 877.99 & 1106.19 & 409.65 & 667.64 & 1328.48 \\
\hline 59 & 336 & 54.83 & 2.27 & 8.95 & 54.94 & 15.1 & 14.06 & 14.3 & 11.48 & 411.40 & 524.45 & 255.24 & 377.64 & 488.28 \\
\hline 60 & 683 & 63.11 & 5.35 & 5.83 & 30.56 & 10.77 & 8.25 & 6.51 & 5.03 & 107.87 & 271.47 & 30.50 & 51.92 & 77.60 \\
\hline 61 & 29.6 & 14.88 & 16.76 & 7.48 & 35.21 & 12.09 & 8.13 & 7.87 & 7.12 & 4.92 & 10.13 & 0.70 & 3.30 & 5.54 \\
\hline 62 & 62.8 & 18.94 & 15.07 & 5.71 & 35.12 & 12.1 & 7.95 & 7.8 & 7.27 & 16.38 & 40.75 & 3.98 & 6.74 & 14.05 \\
\hline 63 & 266 & 31.48 & 8.34 & 3.73 & 38.96 & 13.06 & 9.24 & 8.7 & 7.96 & 136.74 & 221.12 & 61.33 & 143.72 & 120.79 \\
\hline 64 & 295 & 48.56 & 10.13 & 7.99 & 36.14 & 12.19 & 9.02 & 8.12 & 6.81 & 82.34 & 133.47 & 34.79 & 78.61 & 82.50 \\
\hline 65 & 120 & 23.57 & 12.11 & 4.63 & 39.35 & 13.54 & 9.09 & 8.73 & 7.99 & 92.83 & 150.86 & 34.01 & 81.50 & 104.94 \\
\hline 66 & 233 & 33.31 & 6.6 & 4.76 & 39.82 & 12.6 & 8.36 & 10.2 & 8.66 & 239.47 & 336.03 & 37.46 & 303.93 & 280.46 \\
\hline 67 & 142 & 24.06 & 10.77 & 4.08 & 40.11 & 12.09 & 8.61 & 10.55 & 8.86 & 76.29 & 136.85 & 23.36 & 60.76 & 84.19 \\
\hline 68 & 207 & 31.23 & 6.72 & 4.71 & 40.07 & 12.44 & 7.33 & 11.09 & 9.21 & 101.27 & 148.22 & 24.20 & 82.76 & 149.91 \\
\hline 69 & 150 & 28.42 & 8.31 & 5.39 & 40.74 & 11.96 & 8.43 & 11 & 9.35 & 71.25 & 114.35 & 13.51 & 57.70 & 99.43 \\
\hline 70 & 112 & 24.82 & 10.31 & 5.5 & 40.68 & 12.11 & 9.12 & 10.54 & 8.91 & 68.54 & 96.00 & 51.11 & 41.58 & 85.49 \\
\hline
\end{tabular}


Table 1. Basin characteristics for streamflow-gaging stations in or near Texas, with at least 8 years of mean annual

\begin{tabular}{|c|c|c|c|c|c|c|c|c|c|}
\hline $\begin{array}{c}\text { USGS } \\
\text { station } \\
\text { no. }\end{array}$ & $\begin{array}{c}\text { Map } \\
\text { no. } \\
\text { (pl. 1) }\end{array}$ & USGS station name & $\begin{array}{c}\text { Region } \\
\text { no. }\end{array}$ & $\begin{array}{c}\text { Base } \\
\text { station } \\
\text { no. }\end{array}$ & Latitude & Longitude & $\begin{array}{l}\text { First } \\
\text { year } \\
\text { of } \\
\text { record }\end{array}$ & $\begin{array}{l}\text { Last } \\
\text { year } \\
\text { of } \\
\text { record }\end{array}$ & $\begin{array}{l}\text { Weight } \\
\text { factor }\end{array}$ \\
\hline 08065800 & 71 & Bedias Creek near Madisonville, Texas & 8 & 08117500 & $30^{\circ} 53^{\prime} 03^{\prime \prime}$ & $95^{\circ} 46^{\prime} 39^{\prime \prime}$ & 1968 & 1990 & 23 \\
\hline 08066170 & 72 & Kickapoo Creek near Onalaska, Texas & 11 & 08031000 & $30^{\circ} 54^{\prime} 25^{\prime \prime}$ & $95^{\circ} 05^{\prime} 18^{\prime \prime}$ & 1967 & 1990 & 24 \\
\hline 08066200 & 73 & Long King Creek at Livingston, Texas & 11 & 08070000 & $30^{\circ} 42^{\prime} 58^{\prime \prime}$ & $94^{\circ} 57^{\prime} 31^{\prime \prime}$ & 1964 & 1990 & 27 \\
\hline 08066300 & 74 & Menard Creek near Rye, Texas & 11 & 08070000 & $30^{\circ} 28^{\prime} 52^{\prime \prime}$ & $94^{\circ} 46^{\prime} 46^{\prime \prime}$ & 1967 & 1990 & 24 \\
\hline 08066400 & 75 & Big Creek near Shepherd, Texas & 11 & 08070000 & $30^{\circ} 30^{\prime} 59^{\prime \prime}$ & $94^{\circ} 59^{\prime} 06^{\prime \prime}$ & 1967 & 1989 & 23 \\
\hline 08067500 & 76 & Cedar Bayou near Crosby, Texas & 11 & 08031000 & $29^{\circ} 58^{\prime} 20^{\prime \prime}$ & $94^{\circ} 59^{\prime} 10^{\prime \prime}$ & 1972 & 1990 & 19 \\
\hline 08068000 & 77 & West Fork San Jacinto River near Conroe, Texas & 11 & 08068500 & $30^{\circ} 14^{\prime} 40^{\prime \prime}$ & $95^{\circ} 27^{\prime} 25^{\prime \prime}$ & 1961 & 1972 & 12 \\
\hline 08068500 & 78 & Spring Creek near Spring, Texas & 11 & & $30^{\circ} 06^{\prime} 37^{\prime \prime}$ & $95^{\circ} 26^{\prime} 10^{\prime \prime}$ & 1961 & 1990 & 30 \\
\hline 08068520 & 79 & Spring Creek at Spring, Texas & 11 & & $30^{\circ} 05^{\prime} 31^{\prime \prime}$ & $95^{\circ} 24^{\prime} 21^{\prime \prime}$ & 1961 & 1990 & 30 \\
\hline 08068720 & 80 & Cypress Creek at Katy-Hockley Road near Hockley, Texas & 11 & 08078000 & $29^{\circ} 57^{\prime} 00^{\prime \prime}$ & $95^{\circ} 48^{\prime} 29^{\prime \prime}$ & 1976 & 1990 & 13 \\
\hline 08068740 & 81 & Cypress Creek at House-Hahl Road near Cypress, Texas & 11 & 08078000 & $29^{\circ} 57^{\prime} 32^{\prime \prime}$ & $95^{\circ} 43^{\prime} 03^{\prime \prime}$ & 1976 & 1990 & 15 \\
\hline 08068780 & 82 & Little Cypress Creek near Cypress, Texas & 11 & 08078000 & $30^{\circ} 00^{\prime} 57^{\prime \prime}$ & $95^{\circ} 41^{\prime} 50^{\prime \prime}$ & 1983 & 1990 & 8 \\
\hline 08068800 & 83 & Cypress Creek at Grant Road near Cypress, Texas & 11 & 08068500 & $29^{\circ} 58^{\prime} 24 "$ & $95^{\circ} 35^{\prime} 54^{\prime \prime}$ & 1983 & 1990 & 8 \\
\hline 08070000 & 84 & East Fork San Jacinto River near Cleveland, Texas & 11 & & $30^{\circ} 20^{\prime} 11^{\prime \prime}$ & $95^{\circ} 06^{\prime} 14^{\prime \prime}$ & 1961 & 1990 & 30 \\
\hline 08070500 & 85 & Caney Creek near Splendora, Texas & 11 & & $30^{\circ} 15^{\prime} 34^{\prime \prime}$ & $95^{\circ} 18^{\prime} 08^{\prime \prime}$ & 1961 & 1990 & 30 \\
\hline 08071000 & 86 & Peach Creek at Splendora, Texas & 11 & 08070500 & $30^{\circ} 13^{\prime} 57^{\prime \prime}$ & $95^{\circ} 10^{\prime} 05^{\prime \prime}$ & 1961 & 1977 & 17 \\
\hline 08072300 & 87 & Buffalo Bayou near Katy, Texas & 11 & 08115000 & $29^{\circ} 44^{\prime} 35^{\prime \prime}$ & $95^{\circ} 48^{\prime} 24^{\prime \prime}$ & 1978 & 1990 & 13 \\
\hline 08072730 & 88 & Bear Creek near Barker, Texas & 11 & 08115000 & $29^{\circ} 49^{\prime} 50^{\prime \prime}$ & $95^{\circ} 41^{\prime} 12^{\prime \prime}$ & 1978 & 1990 & 13 \\
\hline 08078000 & 89 & Chocolate Bayou near Alvin, Texas & 11 & & $29^{\circ} 22^{\prime} 09^{\prime \prime}$ & $95^{\circ} 19^{\prime} 14^{\prime \prime}$ & 1961 & 1990 & 30 \\
\hline 08079600 & 90 & Double Mountain Fork Brazos River at Justiceburg, Texas & 3 & 08080500 & $33^{\circ} 02^{\prime} 18^{\prime \prime}$ & $101^{\circ} 11^{\prime} 50^{\prime \prime}$ & 1963 & 1990 & 28 \\
\hline 08080500 & 91 & Double Mountain Fork Brazos River near Aspermont, Texas & 3 & & $33^{\circ} 00^{\prime} 29^{\prime \prime}$ & $100^{\circ} 10^{\prime} 49^{\prime \prime}$ & 1961 & 1990 & 30 \\
\hline 08080700 & 92 & Running Water Draw at Plainview, Texas & 1 & 07232500 & $34^{\circ} 10^{\prime} 44^{\prime \prime}$ & $101^{\circ} 42^{\prime} 08^{\prime \prime}$ & 1962 & 1978 & 17 \\
\hline 08081200 & 93 & Croton Creek near Jayton, Texas & 3 & 07311800 & $33^{\circ} 17^{\prime} 18^{\prime \prime}$ & $100^{\circ} 25^{\prime} 52^{\prime \prime}$ & 1961 & 1986 & 26 \\
\hline 08081500 & 94 & Salt Croton Creek near Aspermont, Texas & 3 & 07311800 & $33^{\circ} 24^{\prime} 03^{\prime \prime}$ & $100^{\circ} 24^{\prime} 29^{\prime \prime}$ & 1961 & 1977 & 17 \\
\hline 08082100 & 95 & Stinking Creek near Aspermont, Texas & 3 & 07311800 & $33^{\circ} 14^{\prime} 00^{\prime \prime}$ & $100^{\circ} 12^{\prime} 47^{\prime \prime}$ & 1966 & 1983 & 18 \\
\hline 08082180 & 96 & North Croton Creek near Knox City, Texas & 3 & 07311800 & $33^{\circ} 22^{\prime} 59^{\prime \prime}$ & $100^{\circ} 04^{\prime} 51^{\prime \prime}$ & 1966 & 1986 & 21 \\
\hline 08082500 & 97 & Brazos River at Seymour, Texas & 3 & 08080500 & $33^{\circ} 34^{\prime} 51^{\prime \prime}$ & $99^{\circ} 16^{\prime} 02^{\prime \prime}$ & 1961 & 1973 & 13 \\
\hline 08082700 & 98 & Millers Creek near Munday, Texas & 3 & 08051000 & $33^{\circ} 19^{\prime} 45^{\prime \prime}$ & $99^{\circ} 27^{\prime} 53^{\prime \prime}$ & 1964 & 1990 & 27 \\
\hline 08083100 & 99 & Clear Fork Brazos River near Roby, Texas & 3 & 08080500 & $32^{\circ} 47^{\prime} 15^{\prime \prime}$ & $100^{\circ} 23^{\prime} 18^{\prime \prime}$ & 1963 & 1990 & 28 \\
\hline 08083240 & 100 & Clear Fork Brazos River at Hawley, Texas & 3 & 08080500 & $32^{\circ} 35^{\prime} 53^{\prime \prime}$ & $99^{\circ} 48^{\prime} 53^{\prime \prime}$ & 1968 & 1989 & 22 \\
\hline 08083245 & 101 & Mulberry Creek near Hawley, Texas & 3 & 08128000 & $32^{\circ} 34^{\prime} 04^{\prime \prime}$ & $99^{\circ} 47^{\prime} 32^{\prime \prime}$ & 1969 & 1989 & 21 \\
\hline 08084800 & 102 & California Creek near Stamford, Texas & 3 & 08080500 & $32^{\circ} 55^{\prime} 51^{\prime \prime}$ & $99^{\circ} 38^{\prime} 32^{\prime \prime}$ & 1963 & 1990 & 28 \\
\hline 08086050 & 103 & Deep Creek at Moran, Texas & 3 & 07300500 & $32^{\circ} 33^{\prime} 33^{\prime \prime}$ & $99^{\circ} 10^{\prime} 11^{\prime \prime}$ & 1964 & 1975 & 12 \\
\hline 08086100 & 104 & Hubbard Creek near Albany, Texas & 3 & 07300500 & $32^{\circ} 41^{\prime} 21^{\prime \prime}$ & $99^{\circ} 09^{\prime} 52^{\prime \prime}$ & 1963 & 1975 & 13 \\
\hline 08086150 & 105 & North Fork Hubbard Creek near Albany, Texas & 3 & 08105000 & $32^{\circ} 42^{\prime} 27^{\prime \prime}$ & $99^{\circ} 16^{\prime} 29^{\prime \prime}$ & 1964 & 1990 & 27 \\
\hline
\end{tabular}

Footnote at end of table. 
streamflow data, 1961-90-Continued

\begin{tabular}{|c|c|c|c|c|c|c|c|c|c|c|c|c|c|c|}
\hline \multirow{2}{*}{$\begin{array}{c}\text { Map } \\
\text { no. } \\
\text { (pl. 1) }\end{array}$} & \multirow{2}{*}{$\begin{array}{l}\text { Contri- } \\
\text { buting } \\
\text { drainage } \\
\text { area } \\
(\mathrm{A}) \\
\left(\mathrm{mi}^{2}\right)\end{array}$} & \multirow{2}{*}{$\begin{array}{c}\text { Main } \\
\text { stream } \\
\text { length } \\
(\mathrm{L}) \\
(\mathrm{mi})\end{array}$} & \multirow{2}{*}{$\begin{array}{c}\text { Stream } \\
\text { slope } \\
(\mathrm{SL}) \\
(\mathrm{ft} / \mathrm{mi})\end{array}$} & \multirow{2}{*}{$\begin{array}{c}\text { Basin } \\
\text { shape } \\
\text { factor } \\
\text { (SH) }\end{array}$} & \multicolumn{5}{|c|}{$\begin{array}{l}\text { Precipitation } \\
\quad \text { (in.) }\end{array}$} & \multicolumn{5}{|c|}{$\begin{array}{c}\text { Estimated } 1961-90 \text { streamflow } \\
\left(\mathrm{ft}^{3} / \mathrm{s}\right)\end{array}$} \\
\hline & & & & & Annual & Spring & $\begin{array}{l}\text { Sum- } \\
\text { mer }\end{array}$ & Fall & $\begin{array}{l}\text { Win- } \\
\text { ter }\end{array}$ & Annual & Spring & $\begin{array}{l}\text { Sum- } \\
\text { mer }\end{array}$ & Fall & $\begin{array}{l}\text { Win- } \\
\text { ter }\end{array}$ \\
\hline 71 & 321 & 37.84 & 7.8 & 4.46 & 41.27 & 12.25 & 9.43 & 10.68 & 8.91 & 185.19 & 260.66 & 61.95 & 176.15 & 241.98 \\
\hline 72 & 57 & 12.82 & 12.8 & 2.88 & 45.64 & 12.92 & 10.66 & 11.6 & 10.46 & 42.14 & 57.16 & 11.37 & 40.57 & 59.46 \\
\hline 73 & 141 & 22.07 & 10.7 & 3.46 & 47.69 & 13.47 & 10.93 & 12.32 & 10.97 & 98.97 & 134.82 & 29.14 & 87.19 & 144.71 \\
\hline 74 & 152 & 39.18 & 7.54 & 10.1 & 51.93 & 14.8 & 11.62 & 13.63 & 11.88 & 118.49 & 158.15 & 52.46 & 88.87 & 174.48 \\
\hline 75 & 38.8 & 14.45 & 18.16 & 5.38 & 49.63 & 14.49 & 11.19 & 12.88 & 11.07 & 26.37 & 38.08 & 12.24 & 21.01 & 34.16 \\
\hline 76 & 64.9 & 16.34 & 2.81 & 4.11 & 52.16 & 14.98 & 13.23 & 13.42 & 10.53 & 57.29 & 83.95 & 42.29 & 53.25 & 49.66 \\
\hline 77 & 828 & 56.73 & 5.09 & 3.89 & 44.85 & 12.99 & 10.9 & 11.38 & 9.58 & 478.34 & 596.18 & 174.59 & 480.36 & 662.24 \\
\hline 78 & 409 & 51.18 & 6.15 & 6.4 & 43.49 & 12.46 & 10.86 & 11.24 & 8.93 & 222.28 & 305.23 & 106.71 & 200.22 & 276.96 \\
\hline 79 & 419 & 54.75 & 5.93 & 7.15 & 43.58 & 12.49 & 10.88 & 11.25 & 8.96 & 222.28 & 305.23 & 106.71 & 200.22 & 276.96 \\
\hline 80 & 110 & 25.8 & 5.85 & 6.05 & 41.6 & 12.11 & 10.25 & 10.98 & 8.26 & 47.77 & 65.84 & 23.16 & 34.80 & 67.27 \\
\hline 81 & 131 & 31.72 & 4.96 & 7.68 & 42.41 & 12.16 & 10.43 & 11.24 & 8.58 & 63.25 & 90.86 & 33.41 & 48.24 & 80.48 \\
\hline 82 & 41 & 12.91 & 5.84 & 4.07 & 43.93 & 12.3 & 10.88 & 11.66 & 9.09 & 21.37 & 34.59 & 10.98 & 14.76 & 25.15 \\
\hline 83 & 214 & 41.89 & 5.01 & 8.2 & 43.46 & 12.28 & 10.74 & 11.52 & 8.92 & 97.04 & 145.49 & 44.97 & 67.42 & 130.29 \\
\hline 84 & 325 & 45.22 & 5.51 & 6.29 & 47.38 & 13.9 & 10.98 & 12.26 & 10.24 & 227.66 & 313.70 & 93.81 & 189.02 & 314.12 \\
\hline 85 & 105 & 30.24 & 8.79 & 8.71 & 47.68 & 13.92 & 11.68 & 12.07 & 10.01 & 80.88 & 110.52 & 40.46 & 71.60 & 100.95 \\
\hline 86 & 117 & 30.45 & 9.91 & 7.93 & 49.26 & 14.47 & 11.63 & 12.43 & 10.73 & 80.79 & 99.06 & 43.74 & 67.84 & 112.50 \\
\hline 87 & 63.3 & 11.65 & 4.08 & 2.14 & 40.25 & 11.26 & 10.38 & 10.46 & 8.15 & 42.29 & 44.56 & 41.91 & 41.25 & 41.45 \\
\hline 88 & 21.5 & 10.11 & 4.96 & 4.76 & 43.32 & 11.48 & 11.61 & 11.16 & 9.07 & 15.47 & 18.94 & 11.12 & 15.19 & 16.63 \\
\hline 89 & 87.7 & 17.36 & 2.74 & 3.44 & 48.26 & 12.87 & 15.04 & 11.16 & 9.19 & 111.37 & 138.98 & 141.76 & 75.60 & 89.13 \\
\hline 90 & 244 & 36.99 & 16.67 & 5.61 & 20.26 & 6.96 & 7.64 & 3.4 & 2.26 & 28.92 & 47.05 & 43.68 & 20.54 & 4.41 \\
\hline 91 & 1,864 & 326.26 & 7.41 & 57.11 & 18.43 & 5.91 & 7.68 & 3.02 & 1.82 & 137.04 & 217.46 & 203.72 & 100.20 & 26.77 \\
\hline 92 & 382 & 134.24 & 9.07 & 47.17 & 18.51 & 6.64 & 7.25 & 2.86 & 1.76 & 3.62 & 10.35 & 1.14 & 2.95 & 0.05 \\
\hline 93 & 290 & 62.55 & 18.99 & 13.49 & 22.57 & 7.62 & 8.14 & 3.91 & 2.9 & 14.44 & 23.67 & 19.01 & 11.32 & 3.77 \\
\hline 94 & 64.3 & 14.78 & 23.31 & 3.4 & 22.54 & 7.74 & 7.92 & 3.92 & 2.96 & 5.34 & 7.74 & 8.39 & 3.78 & 1.43 \\
\hline 95 & 88.8 & 24.85 & 10.69 & 6.96 & 23.02 & 7.94 & 7.63 & 4.36 & 3.09 & 5.13 & 9.36 & 4.08 & 5.40 & 1.68 \\
\hline 96 & 251 & 56.74 & 11.85 & 12.83 & 23.77 & 8.19 & 8.13 & 4.26 & 3.19 & 18.65 & 17.08 & 34.33 & 19.84 & 3.35 \\
\hline 97 & 5,972 & 448.91 & 7.4 & 33.74 & 19.51 & 6.35 & 7.75 & 3.31 & 2.1 & 282.89 & 443.07 & 381.21 & 237.65 & 69.63 \\
\hline 98 & 104 & 29.54 & 7 & 8.39 & 26.52 & 8.88 & 8.71 & 5.07 & 3.86 & 7.10 & 13.50 & 9.46 & 3.24 & 2.21 \\
\hline 99 & 228 & 38.86 & 13.09 & 6.62 & 23.86 & 8.1 & 8.41 & 4.44 & 2.91 & 11.11 & 17.47 & 15.53 & 8.30 & 3.14 \\
\hline 100 & 1,416 & 107.32 & 7.37 & 8.13 & 24.28 & 7.76 & 8.7 & 4.61 & 3.21 & 59.99 & 90.03 & 73.37 & 50.56 & 26.02 \\
\hline 101 & 205 & 49.71 & 17.69 & 12.06 & 24.67 & 7.65 & 8.78 & 4.85 & 3.39 & 10.98 & 12.92 & 18.26 & 8.14 & 4.60 \\
\hline 102 & 478 & 64.88 & 7.58 & 8.81 & 24.54 & 8.36 & 8.22 & 4.65 & 3.31 & 37.05 & 57.53 & 48.83 & 27.65 & 14.19 \\
\hline 103 & 228 & 38.25 & 16.47 & 6.42 & 25.05 & 7.93 & 7.2 & 5.63 & 4.29 & 15.44 & 21.64 & 6.79 & 10.53 & 22.79 \\
\hline 104 & 454 & 50.8 & 13.76 & 5.68 & 25.81 & 8.29 & 7.55 & 5.66 & 4.31 & 33.78 & 52.08 & 15.95 & 21.72 & 45.36 \\
\hline 105 & 39.3 & 13.39 & 36.26 & 4.57 & 28.45 & 9.58 & 8.82 & 5.61 & 4.44 & 6.45 & 7.09 & 11.53 & 3.17 & 4.03 \\
\hline
\end{tabular}


Table 1. Basin characteristics for streamflow-gaging stations in or near Texas, with at least 8 years of mean annual

\begin{tabular}{|c|c|c|c|c|c|c|c|c|c|}
\hline $\begin{array}{c}\text { USGS } \\
\text { station } \\
\text { no. }\end{array}$ & $\begin{array}{c}\text { Map } \\
\text { no. } \\
\text { (pl. 1) }\end{array}$ & USGS station name & $\begin{array}{c}\text { Region } \\
\text { no. }\end{array}$ & $\begin{array}{c}\text { Base } \\
\text { station } \\
\text { no. }\end{array}$ & Latitude & Longitude & $\begin{array}{l}\text { First } \\
\text { year } \\
\text { of } \\
\text { record }\end{array}$ & $\begin{array}{l}\text { Last } \\
\text { year } \\
\text { of } \\
\text { record }\end{array}$ & $\begin{array}{l}\text { Weight } \\
\text { factor }\end{array}$ \\
\hline 08086212 & 106 & Hubbard Creek below Albany, Texas & 3 & 07299570 & $32^{\circ} 43^{\prime} 58^{\prime \prime}$ & $99^{\circ} 08^{\prime} 25^{\prime \prime}$ & 1967 & 1990 & 24 \\
\hline 08086260 & 107 & Pecan Creek near Eolian, Texas & 3 & 08017200 & $32^{\circ} 35^{\prime} 01^{\prime \prime}$ & $99^{\circ} 01^{\prime} 57^{\prime \prime}$ & 1967 & 1975 & 9 \\
\hline 08086290 & 108 & Big Sandy Creek above Breckenridge, Texas & 3 & 08101000 & $32^{\circ} 38^{\prime} 54^{\prime \prime}$ & $99^{\circ} 00^{\prime} 15^{\prime \prime}$ & 1976 & 1990 & 15 \\
\hline 08088100 & 109 & Salt Creek at Olney, Texas & 3 & 08061540 & $33^{\circ} 22^{\prime} 13^{\prime \prime}$ & $98^{\circ} 44^{\prime} 40^{\prime \prime}$ & 1961 & 1977 & 17 \\
\hline 08088300 & 110 & Briar Creek near Graham, Texas & 3 & 07315200 & $33^{\circ} 12^{\prime} 43^{\prime \prime}$ & $98^{\circ} 37^{\prime} 06^{\prime \prime}$ & 1961 & 1989 & 29 \\
\hline 08088450 & 111 & Big Cedar Creek near Ivan, Texas & 3 & 07332500 & $32^{\circ} 49^{\prime} 39^{\prime \prime}$ & $98^{\circ} 43^{\prime} 25^{\prime \prime}$ & 1966 & 1989 & 24 \\
\hline 08091500 & 112 & Paluxy River at Glen Rose, Texas & 7 & 08049700 & $32^{\circ} 13^{\prime} 53^{\prime \prime}$ & $97^{\circ} 46^{\prime} 37^{\prime \prime}$ & 1961 & 1982 & 22 \\
\hline 08093250 & 113 & Hackberry Creek at Hillsboro, Texas & 7 & 08049700 & $32^{\circ} 00^{\prime} 20^{\prime \prime}$ & $97^{\circ} 08^{\prime} 59^{\prime \prime}$ & 1980 & 1990 & 11 \\
\hline 08093500 & 114 & Aquilla Creek near Aquilla, Texas & $7^{1}(8)$ & 08049700 & $31^{\circ} 50^{\prime} 40^{\prime \prime}$ & $97^{\circ} 12^{\prime} 04^{\prime \prime}$ & 1961 & 1982 & 22 \\
\hline 08095300 & 115 & Middle Bosque River near McGregor, Texas & $7^{1}(8)$ & 08101000 & $31^{\circ} 30^{\prime} 33^{\prime \prime}$ & $97^{\circ} 21^{\prime} 56^{\prime \prime}$ & 1961 & 1985 & 25 \\
\hline 08095400 & 116 & Hog Creek near Crawford, Texas & $7^{1}(8)$ & 08064700 & $31^{\circ} 33^{\prime} 20^{\prime \prime}$ & $97^{\circ} 21^{\prime} 22^{\prime \prime}$ & 1961 & 1979 & 19 \\
\hline 08098300 & 117 & Little Pond Creek near Burlington, Texas & 8 & 08049700 & $31^{\circ} 01^{\prime} 35^{\prime \prime}$ & $96^{\circ} 59^{\prime} 17^{\prime \prime}$ & 1963 & 1982 & 20 \\
\hline 08099300 & 118 & Sabana River near De Leon, Texas & 4 & 08061540 & $32^{\circ} 06^{\prime} 50^{\prime \prime}$ & $98^{\circ} 36^{\prime} 19^{\prime \prime}$ & 1961 & 1979 & 19 \\
\hline 08101000 & 119 & Cowhouse Creek at Pidcoke, Texas & $4^{1}(8)$ & & $31^{\circ} 17^{\prime} 05^{\prime \prime}$ & $97^{\circ} 53^{\prime} 05^{\prime \prime}$ & 1961 & 1990 & 30 \\
\hline 08103800 & 120 & Lampasas River near Kempner, Texas & $4^{1}(8)$ & 08101000 & $31^{\circ} 04^{\prime} 54^{\prime \prime}$ & $98^{\circ} 00^{\prime} 59^{\prime \prime}$ & 1963 & 1973 & 11 \\
\hline 08103900 & 121 & South Fork Rocky Creek near Briggs, Texas & $4^{1}(8)$ & 08171000 & $30^{\circ} 54^{\prime} 41^{\prime \prime}$ & $98^{\circ} 02^{\prime} 12^{\prime \prime}$ & 1964 & 1990 & 27 \\
\hline 08104000 & 122 & Lampasas River at Youngsport, Texas & $4^{1}(8)$ & 08101000 & $30^{\circ} 57^{\prime} 26^{\prime \prime}$ & $97^{\circ} 42^{\prime} 30^{\prime \prime}$ & 1961 & 1973 & 13 \\
\hline 08104700 & 123 & North Fork San Gabriel River near Georgetown, Texas & 8 & 08171000 & $30^{\circ} 39^{\prime} 42^{\prime \prime}$ & $97^{\circ} 42^{\prime} 40^{\prime}$ & 1969 & 1979 & 11 \\
\hline 08104900 & 124 & South Fork San Gabriel River at Georgetown, Texas & 8 & 08171000 & $30^{\circ} 37^{\prime} 32^{\prime \prime}$ & $97^{\circ} 41^{\prime 2} 7^{\prime \prime}$ & 1969 & 1990 & 22 \\
\hline 08105000 & 125 & San Gabriel River at Georgetown, Texas & 8 & 08171000 & $30^{\circ} 39^{\prime} 14^{\prime \prime}$ & $97^{\circ} 39^{\prime} 18^{\prime \prime}$ & 1961 & 1973 & 13 \\
\hline 08105100 & 126 & Berry Creek near Georgetown, Texas & 8 & 08171000 & $30^{\circ} 41^{\prime} 28^{\prime \prime}$ & $97^{\circ} 39^{\prime} 21^{\prime \prime}$ & 1968 & 1990 & 23 \\
\hline 08105400 & 127 & San Gabriel River near Circleville, Texas & 8 & 08171000 & $30^{\circ} 37^{\prime} 43^{\prime \prime}$ & $97^{\circ} 28^{\prime} 23^{\prime \prime}$ & 1968 & 1976 & 9 \\
\hline 08105700 & 128 & San Gabriel River at Laneport, Texas & 8 & 08171000 & $30^{\circ} 41^{\prime} 39^{\prime \prime}$ & $97^{\circ} 16^{\prime} 43^{\prime \prime}$ & 1966 & 1979 & 14 \\
\hline 08108200 & 129 & North Elm Creek near Cameron, Texas & 8 & 08171000 & $30^{\circ} 55^{\prime} 52^{\prime \prime}$ & $97^{\circ} 01^{\prime} 13^{\prime \prime}$ & 1963 & 1972 & 10 \\
\hline 08109700 & 130 & Middle Yegua Creek near Dime Box, Texas & 8 & 08171000 & $30^{\circ} 20^{\prime} 21^{\prime \prime}$ & $96^{\circ} 54^{\prime} 16^{\prime \prime}$ & 1963 & 1990 & 28 \\
\hline 08109800 & 131 & East Yegua Creek near Dime Box, Texas & 8 & 08171000 & $30^{\circ} 24^{\prime} 26^{\prime \prime}$ & $96^{\circ} 49^{\prime} 02^{\prime \prime}$ & 1963 & 1990 & 28 \\
\hline 08110100 & 132 & Davidson Creek near Lyons, Texas & 8 & 08068500 & $30^{\circ} 25^{\prime} 10^{\prime \prime}$ & $96^{\circ} 32^{\prime} 24^{\prime \prime}$ & 1963 & 1990 & 28 \\
\hline 08110430 & 133 & Big Creek near Freestone, Texas & $7^{1}(8)$ & 08049700 & $31^{\circ} 30^{\prime} 25^{\prime \prime}$ & $96^{\circ} 19^{\prime} 31^{\prime \prime}$ & 1979 & 1990 & 12 \\
\hline 08111700 & 134 & Mill Creek near Bellville, Texas & 11 & 08068500 & $29^{\circ} 52^{\prime} 51^{\prime \prime}$ & $96^{\circ} 12^{\prime} 18^{\prime \prime}$ & 1964 & 1990 & 27 \\
\hline 08115000 & 135 & Big Creek near Needville, Texas & 11 & & $29^{\circ} 28^{\prime} 35^{\prime \prime}$ & $95^{\circ} 48^{\prime} 45^{\prime \prime}$ & 1961 & 1990 & 30 \\
\hline 08116400 & 136 & Dry Creek near Rosenburg, Texas & 11 & 08115000 & $29^{\circ} 30^{\prime} 42^{\prime \prime}$ & $95^{\circ} 44^{\prime} 48^{\prime \prime}$ & 1961 & 1979 & 19 \\
\hline 08117500 & 137 & San Bernard River near Boling, Texas & 11 & & $29^{\circ} 18^{\prime} 48^{\prime \prime}$ & $95^{\circ} 53^{\prime} 37^{\prime \prime}$ & 1961 & 1990 & 30 \\
\hline 08120500 & 138 & Deep Creek near Dunn, Texas & 3 & 08080500 & $32^{\circ} 34^{\prime} 25^{\prime \prime}$ & $100^{\circ} 54^{\prime} 27^{\prime \prime}$ & 1961 & 1986 & 26 \\
\hline 08126500 & 139 & Colorado River at Ballinger, Texas & 4 & 08150000 & $31^{\circ} 43^{\prime} 58^{\prime \prime}$ & $99^{\circ} 57^{\prime} 13^{\prime \prime}$ & 1961 & 1968 & 8 \\
\hline 08127000 & 140 & Elm Creek at Ballinger, Texas & 4 & 08190000 & $31^{\circ} 44^{\prime} 57^{\prime \prime}$ & $99^{\circ} 56^{\prime} 51^{\prime \prime}$ & 1961 & 1981 & 21 \\
\hline
\end{tabular}

Footnote at end of table. 
streamflow data, 1961-90-Continued

\begin{tabular}{|c|c|c|c|c|c|c|c|c|c|c|c|c|c|c|}
\hline \multirow{2}{*}{$\begin{array}{c}\text { Map } \\
\text { no. } \\
\text { (pl. 1) }\end{array}$} & \multirow{2}{*}{$\begin{array}{l}\text { Contri- } \\
\text { buting } \\
\text { drainage } \\
\text { area } \\
(\mathrm{A}) \\
\left(\mathrm{mi}^{2}\right)\end{array}$} & \multirow{2}{*}{$\begin{array}{c}\text { Main } \\
\text { stream } \\
\text { length } \\
(\mathrm{L}) \\
(\mathrm{mi})\end{array}$} & \multirow{2}{*}{$\begin{array}{c}\text { Stream } \\
\text { slope } \\
\text { (SL) } \\
\text { (ft/mi) }\end{array}$} & \multirow{2}{*}{$\begin{array}{c}\text { Basin } \\
\text { shape } \\
\text { factor } \\
\text { (SH) }\end{array}$} & \multicolumn{5}{|c|}{$\begin{array}{l}\text { Precipitation } \\
\text { (in.) }\end{array}$} & \multicolumn{5}{|c|}{$\begin{array}{l}\text { Estimated 1961-90 streamflow } \\
\qquad\left(\mathrm{ft}^{3} / \mathrm{s}\right)\end{array}$} \\
\hline & & & & & Annual & Spring & $\begin{array}{l}\text { Sum- } \\
\text { mer }\end{array}$ & Fall & $\begin{array}{c}\text { Win- } \\
\text { ter }\end{array}$ & Annual & Spring & $\begin{array}{l}\text { Sum- } \\
\text { mer }\end{array}$ & Fall & $\begin{array}{c}\text { Win- } \\
\text { ter }\end{array}$ \\
\hline 106 & 613 & 56.36 & 12.75 & 5.18 & 26.47 & 8.61 & 7.84 & 5.67 & 4.35 & 60.41 & 86.13 & 74.43 & 41.26 & 39.82 \\
\hline 107 & 26.4 & 13.72 & 26.78 & 7.13 & 26.27 & 8.48 & 7.06 & 6.12 & 4.61 & 2.17 & 3.96 & 0.83 & 0.52 & 3.34 \\
\hline 108 & 280 & 88.7 & 8.1 & 28.1 & 26.27 & 8.51 & 7.1 & 6.06 & 4.6 & 23.86 & 28.55 & 9.20 & 40.64 & 17.05 \\
\hline 109 & 11.8 & 4.5 & 13.13 & 1.71 & 28.67 & 10.53 & 7.89 & 5.75 & 4.5 & 5.19 & 14.88 & 1.37 & 2.82 & 1.70 \\
\hline 110 & 24.2 & 12.14 & 15.41 & 6.09 & 30.38 & 10.95 & 8.36 & 6.26 & 4.81 & 4.66 & 9.58 & 3.90 & 3.00 & 2.15 \\
\hline 111 & 97 & 26.06 & 13.47 & 7 & 28.04 & 9.4 & 7.38 & 6.38 & 4.88 & 13.52 & 17.43 & 9.21 & 18.81 & 8.63 \\
\hline 112 & 410 & 57.73 & 13.64 & 8.13 & 30.95 & 10.82 & 7.53 & 6.67 & 5.93 & 56.47 & 119.93 & 17.79 & 34.89 & 53.28 \\
\hline 113 & 57.9 & 14.69 & 9.15 & 3.73 & 35.15 & 11.82 & 7.5 & 8.3 & 7.53 & 32.83 & 64.21 & 8.59 & 28.51 & 30.03 \\
\hline 114 & 308 & 38.85 & 8.19 & 4.9 & 34.85 & 11.8 & 7.48 & 8.2 & 7.37 & 105.21 & 228.43 & 28.74 & 71.00 & 92.67 \\
\hline 115 & 182 & 31.47 & 19.39 & 5.44 & 33.31 & 11.15 & 7.91 & 7.6 & 6.65 & 73.30 & 121.44 & 37.68 & 48.63 & 85.44 \\
\hline 116 & 78.2 & 34.53 & 17.2 & 15.25 & 32.87 & 11.17 & 7.94 & 7.27 & 6.49 & 28.77 & 51.24 & 11.90 & 19.81 & 32.14 \\
\hline 117 & 23 & 13.87 & 12.06 & 8.36 & 34.5 & 11.03 & 7.69 & 8.74 & 7.04 & 12.53 & 24.72 & 2.63 & 10.05 & 12.74 \\
\hline 118 & 264 & 55.41 & 12.37 & 11.63 & 29.24 & 10.43 & 7.46 & 6.15 & 5.2 & 26.59 & 51.30 & 15.16 & 11.15 & 28.75 \\
\hline 119 & 455 & 71.88 & 13.24 & 11.36 & 29.47 & 9.93 & 7.21 & 6.58 & 5.75 & 81.58 & 151.39 & 37.70 & 50.72 & 86.51 \\
\hline 120 & 818 & 65.33 & 13.46 & 5.22 & 29.45 & 9.92 & 7.2 & 6.81 & 5.52 & 121.97 & 222.89 & 43.89 & 59.41 & 161.70 \\
\hline 121 & 33.3 & 11.44 & 36.15 & 3.93 & 30.66 & 10.17 & 7.49 & 7.13 & 5.87 & 10.49 & 18.30 & 4.29 & 5.04 & 14.32 \\
\hline 122 & 1,240 & 101.61 & 10.49 & 8.33 & 30.03 & 10.01 & 7.33 & 6.96 & 5.73 & 199.77 & 378.10 & 65.67 & 108.30 & 247.04 \\
\hline 123 & 248 & 47.7 & 16.3 & 9.17 & 32.15 & 10.77 & 7.75 & 7.42 & 6.21 & 66.66 & 106.57 & 29.84 & 56.59 & 73.62 \\
\hline 124 & 133 & 38.03 & 19.84 & 10.88 & 32.41 & 10.99 & 7.71 & 7.53 & 6.18 & 48.06 & 87.07 & 22.29 & 33.91 & 48.99 \\
\hline 125 & 405 & 52.23 & 16.02 & 6.73 & 32.31 & 10.86 & 7.75 & 7.48 & 6.22 & 142.76 & 227.53 & 40.40 & 117.31 & 185.82 \\
\hline 126 & 83.1 & 28.01 & 16.04 & 9.44 & 33.42 & 10.78 & 8.12 & 7.79 & 6.73 & 24.94 & 39.01 & 9.40 & 17.01 & 34.34 \\
\hline 127 & 599 & 66.06 & 14.6 & 7.29 & 32.74 & 10.88 & 7.84 & 7.63 & 6.39 & 171.31 & 234.08 & 110.62 & 111.24 & 229.31 \\
\hline 128 & 738 & 84.12 & 12.68 & 9.59 & 33.09 & 10.97 & 7.82 & 7.79 & 6.51 & 236.25 & 393.30 & 83.23 & 187.35 & 281.14 \\
\hline 129 & 44.8 & 22.08 & 10.25 & 10.88 & 34.19 & 10.9 & 7.69 & 8.62 & 6.98 & 26.55 & 45.55 & 12.41 & 26.24 & 22.00 \\
\hline 130 & 236 & 41.08 & 6.79 & 7.15 & 34.96 & 11.3 & 7.8 & 8.94 & 6.92 & 51.32 & 92.08 & 9.71 & 46.27 & 57.20 \\
\hline 131 & 244 & 34.84 & 7.25 & 4.97 & 36.88 & 12.02 & 8.12 & 9.19 & 7.55 & 56.27 & 99.78 & 15.64 & 42.32 & 67.34 \\
\hline 132 & 195 & 39.52 & 8.3 & 8.01 & 38.77 & 12.63 & 8.42 & 9.49 & 8.23 & 64.56 & 113.59 & 14.10 & 47.81 & 82.76 \\
\hline 133 & 57.1 & 19 & 7.25 & 6.32 & 40.72 & 11.92 & 8.64 & 10.92 & 9.24 & 43.76 & 49.54 & 15.57 & 56.82 & 53.11 \\
\hline 134 & 376 & 44.62 & 8.01 & 5.3 & 41.13 & 12.63 & 9.54 & 10.43 & 8.53 & 223.31 & 375.53 & 68.51 & 190.41 & 258.78 \\
\hline 135 & 42.8 & 14.47 & 2.83 & 4.89 & 43.53 & 11.9 & 12.56 & 10.81 & 8.26 & 37.19 & 40.72 & 35.26 & 39.87 & 32.90 \\
\hline 136 & 8.65 & 5.83 & 3.38 & 3.93 & 44.11 & 12.02 & 12.87 & 10.83 & 8.39 & 9.77 & 12.49 & 9.34 & 9.52 & 7.74 \\
\hline 137 & 727 & 83.45 & 4.01 & 9.58 & 41.52 & 11.85 & 11.12 & 10.38 & 8.17 & 496.21 & 597.79 & 437.53 & 480.66 & 468.87 \\
\hline 138 & 188 & 45.17 & 14.02 & 10.85 & 21.97 & 7.66 & 7.96 & 3.97 & 2.38 & 11.09 & 22.50 & 16.14 & 3.63 & 2.07 \\
\hline 139 & 6,160 & 195.95 & 4.3 & 6.23 & 21.54 & 7.23 & 7.77 & 3.95 & 2.59 & 60.92 & 105.24 & 91.71 & 32.35 & 14.36 \\
\hline 140 & 450 & 43.96 & 16.49 & 4.29 & 25.72 & 8.84 & 8.2 & 5.05 & 3.63 & 36.82 & 62.38 & 34.96 & 34.48 & 15.47 \\
\hline
\end{tabular}


Table 1. Basin characteristics for streamflow-gaging stations in or near Texas, with at least 8 years of mean annual

\begin{tabular}{|c|c|c|c|c|c|c|c|c|c|}
\hline $\begin{array}{c}\text { USGS } \\
\text { station } \\
\text { no. }\end{array}$ & $\begin{array}{c}\text { Map } \\
\text { no. } \\
\text { (pl. 1) }\end{array}$ & USGS station name & $\begin{array}{c}\text { Region } \\
\text { no. }\end{array}$ & $\begin{array}{c}\text { Base } \\
\text { station } \\
\text { no. }\end{array}$ & Latitude & Longitude & $\begin{array}{l}\text { First } \\
\text { year } \\
\text { of } \\
\text { record }\end{array}$ & $\begin{array}{l}\text { Last } \\
\text { year } \\
\text { of } \\
\text { record }\end{array}$ & $\begin{array}{l}\text { Weight } \\
\text { factor }\end{array}$ \\
\hline 08128000 & 141 & South Concho River at Christoval, Texas & 4 & & $31^{\circ} 11^{\prime} 15^{\prime \prime}$ & $100^{\circ} 30^{\prime} 06^{\prime \prime}$ & 1961 & 1990 & 30 \\
\hline 08128400 & 142 & Middle Concho River above Tankersley, Texas & 4 & 08134000 & $31^{\circ} 25^{\prime} 38^{\prime \prime}$ & $100^{\circ} 42^{\prime} 39^{\prime \prime}$ & 1962 & 1990 & 29 \\
\hline 08129300 & 143 & Spring Creek above Tankersley, Texas & 4 & & $31^{\circ} 19^{\prime} 48^{\prime \prime}$ & $100^{\circ} 38^{\prime} 24^{\prime \prime}$ & 1961 & 1990 & 30 \\
\hline 08130500 & 144 & Dove Creek at Knickerbocker, Texas & 4 & & $31^{\circ} 16^{\prime} 24^{\prime \prime}$ & $100^{\circ} 37^{\prime} 45^{\prime \prime}$ & 1961 & 1990 & 30 \\
\hline 08131400 & 145 & Pecan Creek near San Angelo, Texas & 4 & 08128000 & $31^{\circ} 18^{\prime} 32^{\prime \prime}$ & $100^{\circ} 26^{\prime} 44^{\prime \prime}$ & 1962 & 1986 & 25 \\
\hline 08133500 & 146 & North Concho River at Sterling City, Texas & 4 & 08134000 & $31^{\circ} 49^{\prime} 48^{\prime \prime}$ & $100^{\circ} 59^{\prime} 36^{\prime \prime}$ & 1961 & 1985 & 25 \\
\hline 08134000 & 147 & North Concho River near Carlsbad, Texas & 4 & & $31^{\circ} 35^{\prime} 33^{\prime \prime}$ & $100^{\circ} 38^{\prime} 12^{\prime \prime}$ & 1961 & 1990 & 30 \\
\hline 08144500 & 148 & San Saba River at Menard, Texas & 4 & & $30^{\circ} 55^{\prime} 08^{\prime \prime}$ & $99^{\circ} 47^{\prime} 07^{\prime \prime}$ & 1961 & 1990 & 30 \\
\hline 08144600 & 149 & San Saba River near Brady, Texas & 4 & 08144500 & $31^{\circ} 00^{\prime} 14^{\prime \prime}$ & $99^{\circ} 16^{\prime} 07^{\prime \prime}$ & 1980 & 1990 & 11 \\
\hline 08148500 & 150 & North Llano River near Junction, Texas & 4 & 08150000 & $30^{\circ} 31^{\prime} 06^{\prime \prime}$ & $99^{\circ} 48^{\prime} 39^{\prime \prime}$ & 1961 & 1977 & 17 \\
\hline 08150000 & 151 & Llano River near Junction, Texas & 4 & & $30^{\circ} 29^{\prime} 51^{\prime \prime}$ & $99^{\circ} 43^{\prime} 19^{\prime \prime}$ & 1961 & 1990 & 30 \\
\hline 08150700 & 152 & Llano River near Mason, Texas & 4 & 08150000 & $30^{\circ} 39^{\prime} 38^{\prime \prime}$ & $99^{\circ} 06^{\prime} 32^{\prime \prime}$ & 1969 & 1990 & 22 \\
\hline 08150800 & 153 & Beaver Creek near Mason, Texas & 4 & 08101000 & $30^{\circ} 38^{\prime} 36^{\prime \prime}$ & $99^{\circ} 05^{\prime} 44^{\prime \prime}$ & 1964 & 1990 & 27 \\
\hline 08151500 & 154 & Llano River at Llano, Texas & 4 & & $30^{\circ} 45^{\prime} 04^{\prime \prime}$ & $98^{\circ} 40^{\prime} 10^{\prime \prime}$ & 1961 & 1990 & 30 \\
\hline 08152000 & 155 & Sandy Creek near Kingsland, Texas & 4 & 08153500 & $30^{\circ} 33^{\prime} 30^{\prime \prime}$ & $98^{\circ} 28^{\prime} 19^{\prime \prime}$ & 1967 & 1990 & 24 \\
\hline 08152900 & 156 & Pedernales River near Fredericksburg, Texas & 5 & 08153500 & $30^{\circ} 13^{\prime} 13^{\prime \prime}$ & $98^{\circ} 52^{\prime} 10^{\prime \prime}$ & 1980 & 1990 & 11 \\
\hline 08153500 & 157 & Pedernales River near Johnson City, Texas & 5 & & $30^{\circ} 17^{\prime} 30^{\prime \prime}$ & $98^{\circ} 23^{\prime} 57^{\prime \prime}$ & 1961 & 1990 & 30 \\
\hline 08154700 & 158 & Bull Creek at Loop 360 near Austin, Texas & 5 & 08115000 & $30^{\circ} 22^{\prime} 19^{\prime \prime}$ & $97^{\circ} 47^{\prime} 04^{\prime \prime}$ & 1979 & 1990 & 12 \\
\hline 08155300 & 159 & Barton Creek at Loop 360, Austin, Texas & 5 & 08200000 & $30^{\circ} 14^{\prime} 40^{\prime \prime}$ & $97^{\circ} 48^{\prime} 07^{\prime \prime}$ & 1978 & 1990 & 13 \\
\hline 08158700 & 160 & Onion Creek near Driftwood, Texas & 5 & 08171000 & $30^{\circ} 04^{\prime} 59^{\prime \prime}$ & $98^{\circ} 00^{\prime} 29^{\prime \prime}$ & 1980 & 1990 & 11 \\
\hline 08158810 & 161 & Bear Creek below Farm Road 1826 near Driftwood, Texas & 5 & 08200000 & $30^{\circ} 09^{\prime} 19^{\prime \prime}$ & $97^{\circ} 56^{\prime} 23^{\prime \prime}$ & 1980 & 1990 & 11 \\
\hline 08158840 & 162 & Slaughter Creek at Farm Road 1826 near Austin, Texas & 5 & 08200000 & $30^{\circ} 12^{\prime} 32^{\prime \prime}$ & $97^{\circ} 54^{\prime} 11^{\prime \prime}$ & 1979 & 1990 & 12 \\
\hline 08160800 & 163 & Redgate Creek near Columbus, Texas & 9 & 08115000 & $29^{\circ} 47^{\prime} 56^{\prime \prime}$ & $96^{\circ} 31^{\prime} 55^{\prime \prime}$ & 1963 & 1990 & 28 \\
\hline 08162600 & 164 & Tres Palacios River near Midfield, Texas & 9 & 08078000 & $28^{\circ} 55^{\prime} 40^{\prime \prime}$ & $96^{\circ} 10^{\prime} 15^{\prime \prime}$ & 1971 & 1990 & 20 \\
\hline 08163500 & 165 & Lavaca River at Hallettsville, Texas & 9 & & $29^{\circ} 26^{\prime} 35^{\prime \prime}$ & $96^{\circ} 56^{\prime} 39^{\prime \prime}$ & 1961 & 1990 & 30 \\
\hline 08164000 & 166 & Lavaca River near Edna, Texas & 9 & & $28^{\circ} 57^{\prime} 35^{\prime \prime}$ & $96^{\circ} 41^{\prime} 10^{\prime \prime}$ & 1961 & 1990 & 30 \\
\hline 08164300 & 167 & Navidad River near Hallettsville, Texas & 9 & 08164000 & $29^{\circ} 28^{\prime} 00^{\prime \prime}$ & $96^{\circ} 48^{\prime} 45^{\prime \prime}$ & 1962 & 1990 & 29 \\
\hline 08164350 & 168 & Navidad River near Speaks, Texas & 9 & 08164000 & $29^{\circ} 19^{\prime} 18^{\prime \prime}$ & $96^{\circ} 42^{\prime} 32^{\prime \prime}$ & 1982 & 1989 & 8 \\
\hline 08164450 & 169 & Sandy Creek near Louise, Texas & 9 & 08164000 & $29^{\circ} 09^{\prime} 34^{\prime \prime}$ & $96^{\circ} 32^{\prime} 47^{\prime \prime}$ & 1978 & 1990 & 13 \\
\hline 08164500 & 170 & Navidad River near Ganado, Texas & 9 & 08164000 & $29^{\circ} 01^{\prime} 32^{\prime \prime}$ & $96^{\circ} 33^{\prime} 08^{\prime \prime}$ & 1961 & 1979 & 19 \\
\hline 08164503 & 171 & West Mustang Creek near Ganado, Texas & 9 & 08164000 & $29^{\circ} 04^{\prime} 17^{\prime \prime}$ & $96^{\circ} 28^{\prime} 01^{\prime \prime}$ & 1978 & 1990 & 13 \\
\hline 08164600 & 172 & Garcitas Creek near Inez, Texas & 9 & 08164000 & $28^{\circ} 53^{\prime} 28^{\prime \prime}$ & $96^{\circ} 49^{\prime} 08^{\prime \prime}$ & 1971 & 1990 & 20 \\
\hline 08164800 & 173 & Placedo Creek near Placedo, Texas & $9^{1}(6)$ & 08164000 & $28^{\circ} 43^{\prime} 30^{\prime \prime}$ & $96^{\circ} 46^{\prime} 07^{\prime \prime}$ & 1971 & 1990 & 20 \\
\hline 08165300 & 174 & North Fork Guadalupe River near Hunt, Texas & 5 & 08167000 & $30^{\circ} 03^{\prime} 36^{\prime \prime}$ & $99^{\circ} 23^{\prime} 40^{\prime \prime}$ & 1968 & 1990 & 23 \\
\hline 08165500 & 175 & Guadalupe River at Hunt, Texas & 5 & 08167000 & $30^{\circ} 04^{\prime} 08^{\prime \prime}$ & $99^{\circ} 19^{\prime} 23^{\prime \prime}$ & 1966 & 1990 & 25 \\
\hline
\end{tabular}

Footnote at end of table. 
streamflow data, 1961-90-Continued

\begin{tabular}{|c|c|c|c|c|c|c|c|c|c|c|c|c|c|c|}
\hline \multirow{2}{*}{$\begin{array}{c}\text { Map } \\
\text { no. } \\
\text { (pl. 1) }\end{array}$} & \multirow{2}{*}{$\begin{array}{l}\text { Contri- } \\
\text { buting } \\
\text { drainage } \\
\text { area } \\
(\mathrm{A}) \\
\left(\mathrm{mi}^{2}\right)\end{array}$} & \multirow{2}{*}{$\begin{array}{c}\text { Main } \\
\text { stream } \\
\text { length } \\
\text { (L) } \\
\text { (mi) }\end{array}$} & \multirow{2}{*}{$\begin{array}{c}\text { Stream } \\
\text { slope } \\
(\mathrm{SL}) \\
(\mathrm{ft} / \mathrm{mi})\end{array}$} & \multirow{2}{*}{$\begin{array}{c}\text { Basin } \\
\text { shape } \\
\text { factor } \\
\text { (SH) }\end{array}$} & \multicolumn{5}{|c|}{$\begin{array}{l}\text { Precipitation } \\
\text { (in.) }\end{array}$} & \multicolumn{5}{|c|}{$\begin{array}{l}\text { Estimated 1961-90 streamflow } \\
\qquad\left(\mathrm{ft}^{3} / \mathrm{s}\right)\end{array}$} \\
\hline & & & & & Annual & Spring & $\begin{array}{l}\text { Sum- } \\
\text { mer }\end{array}$ & Fall & $\begin{array}{c}\text { Win- } \\
\text { ter }\end{array}$ & Annual & Spring & $\begin{array}{c}\text { Sum- } \\
\text { mer }\end{array}$ & Fall & $\begin{array}{c}\text { Win- } \\
\text { ter }\end{array}$ \\
\hline 141 & 354 & 33.85 & 13.37 & 3.24 & 21.27 & 6.88 & 7.1 & 4.35 & 2.94 & 25.52 & 25.16 & 26.69 & 28.90 & 21.32 \\
\hline 142 & 1,611 & 102.12 & 7.61 & 6.47 & 18.83 & 5.74 & 7.13 & 3.71 & 2.25 & 18.50 & 21.44 & 27.90 & 15.19 & 9.46 \\
\hline 143 & 405 & 54.5 & 13.36 & 7.33 & 20.83 & 6.71 & 7.17 & 4.1 & 2.85 & 13.36 & 14.36 & 16.81 & 12.05 & 10.22 \\
\hline 144 & 198 & 33.72 & 15.66 & 5.74 & 21.35 & 6.94 & 7.22 & 4.22 & 2.97 & 16.57 & 19.63 & 16.01 & 17.20 & 13.42 \\
\hline 145 & 81.1 & 19.76 & 23.24 & 4.82 & 21.01 & 7.1 & 6.62 & 4.35 & 2.94 & 2.72 & 1.94 & 5.55 & 2.16 & 1.23 \\
\hline 146 & 568 & 48.27 & 10.6 & 4.1 & 19.25 & 6.07 & 7.48 & 3.41 & 2.29 & 5.19 & 7.04 & 9.87 & 1.64 & 2.22 \\
\hline 147 & 1,191 & 83.8 & 9.44 & 5.9 & 19.9 & 6.37 & 7.42 & 3.68 & 2.43 & 12.31 & 13.66 & 23.48 & 8.17 & 3.92 \\
\hline 148 & 1,128 & 58.72 & 9.55 & 3.06 & 23.11 & 7.39 & 7.66 & 4.61 & 3.45 & 51.73 & 38.11 & 92.59 & 44.78 & 31.46 \\
\hline 149 & 1,626 & 100.26 & 8.87 & 6.18 & 23.75 & 7.67 & 7.75 & 4.72 & 3.61 & 83.67 & 78.45 & 100.80 & 96.58 & 58.86 \\
\hline 150 & 914 & 54.71 & 11.87 & 3.27 & 23.4 & 7.71 & 7.49 & 4.83 & 3.37 & 80.76 & 87.03 & 121.48 & 68.51 & 46.01 \\
\hline 151 & 1,851 & 74.29 & 9.98 & 2.98 & 24.03 & 7.89 & 7.71 & 4.97 & 3.46 & 201.04 & 213.93 & 237.10 & 210.10 & 143.01 \\
\hline 152 & 3,242 & 128.04 & 8.79 & 5.06 & 24.74 & 8.24 & 7.67 & 5.14 & 3.69 & 298.84 & 338.25 & 345.13 & 305.96 & 206.01 \\
\hline 153 & 215 & 33.9 & 25.74 & 5.35 & 27.45 & 9.18 & 7.82 & 6.15 & 4.3 & 16.34 & 24.38 & 13.29 & 14.14 & 13.53 \\
\hline 154 & 4,192 & 159.74 & 8.24 & 6.09 & 25.29 & 8.45 & 7.65 & 5.34 & 3.85 & 397.60 & 480.97 & 400.47 & 389.01 & 319.96 \\
\hline 155 & 346 & 48.34 & 24.84 & 6.75 & 29.38 & 10 & 7.95 & 6.6 & 4.83 & 54.47 & 91.96 & 24.59 & 45.79 & 55.54 \\
\hline 156 & 369 & 38.86 & 17.64 & 4.09 & 28.59 & 9.28 & 8.32 & 6.54 & 4.45 & 50.28 & 80.22 & 35.95 & 46.69 & 38.28 \\
\hline 157 & 901 & 79.69 & 14.57 & 7.05 & 29.9 & 10.06 & 8.19 & 6.82 & 4.83 & 192.02 & 326.10 & 129.36 & 160.09 & 152.53 \\
\hline 158 & 22.3 & 9.4 & 47.82 & 3.96 & 31.63 & 11 & 7.41 & 7.49 & 5.73 & 10.55 & 11.82 & 3.95 & 16.75 & 9.71 \\
\hline 159 & 116 & 43.53 & 18.84 & 16.33 & 31.74 & 10.94 & 7.59 & 7.6 & 5.61 & 53.71 & 108.13 & 5.84 & 46.94 & 53.92 \\
\hline 160 & 124 & 31.99 & 21.02 & 8.25 & 33.05 & 11.13 & 8.03 & 8.02 & 5.87 & 50.85 & 85.80 & 15.06 & 41.09 & 61.45 \\
\hline 161 & 12.2 & 5.49 & 55.55 & 2.47 & 32.48 & 11.16 & 7.76 & 7.83 & 5.73 & 4.32 & 6.48 & 1.62 & 4.78 & 4.39 \\
\hline 162 & 8.24 & 4.2 & 52.32 & 2.14 & 31.94 & 11.06 & 7.59 & 7.66 & 5.63 & 3.85 & 5.96 & 1.06 & 4.74 & 3.63 \\
\hline 163 & 17.3 & 7.61 & 18.11 & 3.35 & 41.66 & 12.75 & 10.29 & 9.93 & 8.69 & 5.23 & 8.94 & 1.96 & 4.08 & 5.92 \\
\hline 164 & 145 & 30.55 & 3.33 & 6.44 & 43.28 & 11.89 & 13.5 & 10.19 & 7.7 & 128.15 & 126.25 & 146.41 & 132.28 & 107.68 \\
\hline 165 & 108 & 25.23 & 12.18 & 5.89 & 37.56 & 12.4 & 9.48 & 8.51 & 7.17 & 55.18 & 94.83 & 49.39 & 40.78 & 35.72 \\
\hline 166 & 817 & 85.59 & 5.44 & 8.97 & 38.21 & 12.43 & 10.2 & 8.59 & 6.99 & 365.55 & 631.89 & 238.25 & 295.83 & 296.21 \\
\hline 167 & 332 & 37.31 & 8.35 & 4.19 & 38.32 & 12.31 & 9.74 & 8.87 & 7.4 & 143.43 & 247.28 & 87.01 & 111.36 & 128.08 \\
\hline 168 & 437 & 53.39 & 6.27 & 6.52 & 38.78 & 12.39 & 9.97 & 8.94 & 7.48 & 194.66 & 156.01 & 255.67 & 132.95 & 233.98 \\
\hline 169 & 289 & 55.48 & 5.97 & 10.65 & 40.99 & 12.5 & 11.12 & 9.4 & 7.97 & 179.81 & 244.18 & 134.50 & 148.75 & 191.80 \\
\hline 170 & 826 & 92.67 & 4.78 & 10.4 & 40 & 12.53 & 10.69 & 9.2 & 7.58 & 589.69 & 960.01 & 473.63 & 421.68 & 503.42 \\
\hline 171 & 178 & 39.05 & 2.77 & 8.56 & 41.52 & 12.22 & 11.74 & 9.63 & 7.93 & 109.15 & 136.96 & 100.94 & 104.62 & 94.07 \\
\hline 172 & 91.7 & 24.98 & 6.83 & 6.81 & 37.17 & 11.93 & 11.38 & 7.97 & 5.89 & 45.95 & 79.94 & 37.10 & 35.69 & 31.09 \\
\hline 173 & 68.3 & 21.57 & 4.72 & 6.81 & 38.61 & 11.83 & 12.49 & 8.39 & 5.9 & 53.55 & 64.39 & 70.98 & 48.40 & 30.44 \\
\hline 174 & 168 & 27.57 & 20.35 & 4.53 & 28.44 & 8.89 & 8.94 & 6.34 & 4.27 & 36.01 & 36.92 & 41.73 & 41.84 & 23.56 \\
\hline 175 & 288 & 33.85 & 19.1 & 3.98 & 28.68 & 8.96 & 8.98 & 6.39 & 4.35 & 70.67 & 73.77 & 85.95 & 71.98 & 50.96 \\
\hline
\end{tabular}


Table 1. Basin characteristics for streamflow-gaging stations in or near Texas, with at least 8 years of mean annual

\begin{tabular}{|c|c|c|c|c|c|c|c|c|c|}
\hline $\begin{array}{c}\text { USGS } \\
\text { station } \\
\text { no. }\end{array}$ & $\begin{array}{c}\text { Map } \\
\text { no. } \\
\text { (pl. 1) }\end{array}$ & USGS station name & $\begin{array}{c}\text { Region } \\
\text { no. }\end{array}$ & $\begin{array}{c}\text { Base } \\
\text { station } \\
\text { no. }\end{array}$ & Latitude & Longitude & $\begin{array}{l}\text { First } \\
\text { year } \\
\text { of } \\
\text { record }\end{array}$ & $\begin{array}{l}\text { Last } \\
\text { year } \\
\text { of } \\
\text { record }\end{array}$ & $\begin{array}{c}\text { Weight } \\
\text { factor }\end{array}$ \\
\hline 08166000 & 176 & Johnson Creek near Ingram, Texas & 5 & 08167000 & $30^{\circ} 06^{\prime} 00^{\prime \prime}$ & $99^{\circ} 16 ' 58^{\prime \prime}$ & 1962 & 1990 & 29 \\
\hline 08167000 & 177 & Guadalupe River at Comfort, Texas & 5 & & $29^{\circ} 58^{\prime} 10^{\prime \prime}$ & $98^{\circ} 53^{\prime} 33^{\prime \prime}$ & 1961 & 1990 & 30 \\
\hline 08167500 & 178 & Guadalupe River near Spring Branch, Texas & 5 & & $29^{\circ} 51^{\prime} 38^{\prime \prime}$ & $98^{\circ} 22^{\prime} 58^{\prime \prime}$ & 1961 & 1990 & 30 \\
\hline 08167600 & 179 & Rebecca Creek near Spring Branch, Texas & 5 & 08103900 & $29^{\circ} 55^{\prime} 06^{\prime \prime}$ & $98^{\circ} 22^{\prime} 10^{\prime \prime}$ & 1961 & 1973 & 13 \\
\hline 08171000 & 180 & Blanco River at Wimberley, Texas & 5 & & $29^{\circ} 59^{\prime} 39^{\prime \prime}$ & $98^{\circ} 05^{\prime} 19^{\prime \prime}$ & 1961 & 1990 & 30 \\
\hline 08172000 & 181 & San Marcos River at Luling, Texas & $9^{1}(6)$ & 08171000 & $29^{\circ} 39^{\prime} 54^{\prime \prime}$ & $97^{\circ} 38^{\prime} 59^{\prime \prime}$ & 1961 & 1983 & 23 \\
\hline 08174600 & 182 & Peach Creek below Dilworth, Texas & $9^{1}(6)$ & 08163500 & $29^{\circ} 28^{\prime} 26^{\prime \prime}$ & $97^{\circ} 18^{\prime} 59^{\prime \prime}$ & 1961 & 1979 & 19 \\
\hline 08175000 & 183 & Sandies Creek near Westhoff, Texas & $9^{1}(6)$ & & $29^{\circ} 12^{\prime} 54^{\prime \prime}$ & $97^{\circ} 26{ }^{\prime} 57^{\prime \prime}$ & 1961 & 1990 & 30 \\
\hline 08176900 & 184 & Coleto Creek at Arnold Road near Schroeder, Texas & $9^{1}(6)$ & 08164000 & $28^{\circ} 51^{\prime} 41^{\prime \prime}$ & $97^{\circ} 13^{\prime} 34^{\prime \prime}$ & 1979 & 1990 & 12 \\
\hline 08177000 & 185 & Coleto Creek near Schroeder, Texas & $9^{1}(6)$ & 08175000 & $28^{\circ} 49^{\prime} 53^{\prime \prime}$ & $97^{\circ} 11^{\prime} 10^{\prime \prime}$ & 1961 & 1979 & 19 \\
\hline 08177300 & 186 & Perdido Creek at Farm Road 622 near Fannin, Texas & $9^{1}(6)$ & 08163500 & $28^{\circ} 45^{\prime} 05^{\prime \prime}$ & $97^{\circ} 19^{\prime} 01^{\prime \prime}$ & 1979 & 1990 & 12 \\
\hline 08178880 & 187 & Medina River at Bandera, Texas & 5 & 08200000 & $29^{\circ} 43^{\prime} 26^{\prime \prime}$ & $99^{\circ} 04^{\prime} 13^{\prime \prime}$ & 1983 & 1990 & 8 \\
\hline 08179000 & 188 & Medina River near Pipe Creek, Texas & 5 & 08167000 & $29^{\circ} 40^{\prime} 31^{\prime \prime}$ & $98^{\circ} 58^{\prime} 33^{\prime \prime}$ & 1961 & 1982 & 22 \\
\hline 08179100 & 189 & Red Bluff Creek near Pipe Creek, Texas & 5 & 08200000 & $29^{\circ} 40^{\prime} 51^{\prime \prime}$ & $98^{\circ} 57^{\prime} 19^{\prime \prime}$ & 1961 & 1981 & 21 \\
\hline 08181400 & 190 & Helotes Creek at Helotes, Texas & 5 & 08200000 & $29^{\circ} 34^{\prime} 42^{\prime \prime}$ & $98^{\circ} 41^{\prime} 29^{\prime \prime}$ & 1969 & 1990 & 22 \\
\hline 08183900 & 191 & Cibolo Creek near Boerne, Texas & 5 & 08171000 & $29^{\circ} 46^{\prime} 26^{\prime \prime}$ & $98^{\circ} 41^{\prime} 50^{\prime \prime}$ & 1963 & 1977 & 15 \\
\hline 08185000 & 192 & Cibolo Creek at Selma, Texas & 5 & 08186000 & $29^{\circ} 35^{\prime} 38^{\prime \prime}$ & $98^{\circ} 18^{\prime} 39^{\prime \prime}$ & 1961 & 1980 & 20 \\
\hline 08186000 & 193 & Cibolo Creek near Falls City, Texas & $9^{1}(6)$ & & $29^{\circ} 00^{\prime} 50^{\prime \prime}$ & $97^{\circ} 55^{\prime} 48^{\prime \prime}$ & 1961 & 1990 & 30 \\
\hline 08186500 & 194 & Ecleto Creek near Runge, Texas & $9^{1}(6)$ & 08175000 & $28^{\circ} 55^{\prime} 12^{\prime \prime}$ & $97^{\circ} 46^{\prime} 19^{\prime \prime}$ & 1963 & 1989 & 27 \\
\hline 08189200 & 195 & Copano Creek near Refugio, Texas & $9^{1}(6)$ & 08189500 & $28^{\circ} 18^{\prime} 12^{\prime \prime}$ & $97^{\circ} 06^{\prime} 44^{\prime \prime}$ & 1971 & 1990 & 20 \\
\hline 08189300 & 196 & Medio Creek near Beeville, Texas & $9^{1}(6)$ & 08189500 & $28^{\circ} 28^{\prime} 58^{\prime \prime}$ & $97^{\circ} 39^{\prime} 23^{\prime \prime}$ & 1963 & 1977 & 15 \\
\hline 08189500 & 197 & Misson River at Refugio, Texas & $9^{1}(6)$ & & $28^{\circ} 17^{\prime} 30^{\prime \prime}$ & $97^{\circ} 16^{\prime} 44^{\prime \prime}$ & 1961 & 1990 & 30 \\
\hline 08189700 & 198 & Aransas River near Skidmore, Texas & $9^{1}(6)$ & 08189500 & $28^{\circ} 16^{\prime} 56^{\prime \prime}$ & $97^{\circ} 37^{\prime} 14^{\prime \prime}$ & 1965 & 1990 & 26 \\
\hline 08189800 & 199 & Chiltipin Creek at Sinton, Texas & $9^{1}(6)$ & 08189500 & $28^{\circ} 02^{\prime} 48^{\prime \prime}$ & $97^{\circ} 30^{\prime} 13^{\prime \prime}$ & 1971 & 1990 & 20 \\
\hline 08190000 & 200 & Nueces River at Laguna, Texas & 5 & & $29^{\circ} 25^{\prime} 42^{\prime \prime}$ & $99^{\circ} 59^{\prime} 49^{\prime \prime}$ & 1961 & 1990 & 30 \\
\hline 08190500 & 201 & West Nueces River near Brackettville, Texas & 5 & & $29^{\circ} 28^{\prime} 21^{\prime \prime}$ & $100^{\circ} 14^{\prime} 10^{\prime \prime}$ & 1961 & 1990 & 30 \\
\hline 08194200 & 202 & San Casimiro Creek near Freer, Texas & 6 & 08189500 & $27^{\circ} 57^{\prime} 53^{\prime \prime}$ & $98^{\circ} 58^{\prime} 00^{\prime \prime}$ & 1963 & 1990 & 28 \\
\hline 08194600 & 203 & Nueces River at Simmons, Texas & 6 & 08189500 & $28^{\circ} 25^{\prime} 16^{\prime \prime}$ & $98^{\circ} 17^{\prime} 03^{\prime \prime}$ & 1966 & 1977 & 12 \\
\hline 08195000 & 204 & Frio River at Concan, Texas & 5 & & $29^{\circ} 29^{\prime} 18^{\prime \prime}$ & $99^{\circ} 42^{\prime} 16^{\prime \prime}$ & 1961 & 1990 & 30 \\
\hline 08196000 & 205 & Dry Frio River near Reagan Wells, Texas & 5 & & $29^{\circ} 30^{\prime} 16^{\prime \prime}$ & $99^{\circ} 46^{\prime} 52^{\prime \prime}$ & 1961 & 1990 & 30 \\
\hline 08198000 & 206 & Sabinal River near Sabinal, Texas & 5 & & $29^{\circ} 29^{\prime} 35^{\prime \prime}$ & $99^{\circ} 29^{\prime} 49^{\prime \prime}$ & 1961 & 1990 & 30 \\
\hline 08200000 & 207 & Hondo Creek near Tarpley, Texas & 5 & & $29^{\circ} 34^{\prime} 10^{\prime \prime}$ & $99^{\circ} 14^{\prime} 47^{\prime \prime}$ & 1961 & 1990 & 30 \\
\hline 08201500 & 208 & Seco Creek at Miller Ranch near Utopia, Texas & 5 & 08200000 & $29^{\circ} 34^{\prime} 23^{\prime \prime}$ & $99^{\circ} 24^{\prime} 10^{\prime \prime}$ & 1962 & 1990 & 29 \\
\hline 08205500 & 209 & Frio River near Derby, Texas & 6 & & $28^{\circ} 44^{\prime} 11^{\prime \prime}$ & $99^{\circ} 08^{\prime} 40^{\prime \prime}$ & 1961 & 1990 & 30 \\
\hline 08206600 & 210 & Frio River at Tilden, Texas & 6 & 08205500 & $28^{\circ} 28^{\prime} 02^{\prime \prime}$ & $98^{\circ} 32^{\prime} 50^{\prime \prime}$ & 1979 & 1990 & 12 \\
\hline
\end{tabular}

Footnote at end of table. 
streamflow data, 1961-90-Continued

\begin{tabular}{|c|c|c|c|c|c|c|c|c|c|c|c|c|c|c|}
\hline \multirow{2}{*}{$\begin{array}{c}\text { Map } \\
\text { no. } \\
\text { (pl. 1) }\end{array}$} & \multirow{2}{*}{$\begin{array}{l}\text { Contri- } \\
\text { buting } \\
\text { drainage } \\
\text { area } \\
(\mathrm{A}) \\
\left(\mathrm{mi}^{2}\right)\end{array}$} & \multirow{2}{*}{$\begin{array}{c}\text { Main } \\
\text { stream } \\
\text { length } \\
(\mathrm{L}) \\
(\mathrm{mi})\end{array}$} & \multirow{2}{*}{$\begin{array}{c}\text { Stream } \\
\text { slope } \\
(\mathrm{SL}) \\
(\mathrm{ft} / \mathrm{mi})\end{array}$} & \multirow{2}{*}{$\begin{array}{c}\text { Basin } \\
\text { shape } \\
\text { factor } \\
\text { (SH) }\end{array}$} & \multicolumn{5}{|c|}{$\begin{array}{l}\text { Precipitation } \\
\text { (in.) }\end{array}$} & \multicolumn{5}{|c|}{$\begin{array}{c}\text { Estimated } 1961-90 \text { streamflow } \\
\left(\mathrm{ft}^{3} / \mathrm{s}\right)\end{array}$} \\
\hline & & & & & Annual & Spring & $\begin{array}{l}\text { Sum- } \\
\text { mer }\end{array}$ & Fall & $\begin{array}{l}\text { Win- } \\
\text { ter }\end{array}$ & Annual & Spring & $\begin{array}{c}\text { Sum- } \\
\text { mer }\end{array}$ & Fall & $\begin{array}{c}\text { Win- } \\
\text { ter }\end{array}$ \\
\hline 176 & 114 & 19.8 & 25.03 & 3.44 & 27.83 & 8.73 & 8.67 & 6.08 & 4.35 & 28.37 & 28.32 & 34.02 & 29.31 & 21.83 \\
\hline 177 & 839 & 68.87 & 15.01 & 5.65 & 29.56 & 9.2 & 9.02 & 6.65 & 4.69 & 247.00 & 293.03 & 253.98 & 239.30 & 201.70 \\
\hline 178 & 1,315 & 131.27 & 10.85 & 13.1 & 30.94 & 9.8 & 8.98 & 7.08 & 5.08 & 413.65 & 555.88 & 371.23 & 362.04 & 365.46 \\
\hline 179 & 10.9 & 5.57 & 77.79 & 2.84 & 33.44 & 11.09 & 8.52 & 7.9 & 5.93 & 5.49 & 10.53 & 1.71 & 3.62 & 6.10 \\
\hline 180 & 355 & 60.39 & 19.02 & 10.27 & 33.72 & 11.09 & 8.43 & 8.08 & 6.12 & 147.65 & 223.43 & 87.12 & 123.68 & 156.39 \\
\hline 181 & 838 & 124.26 & 13.07 & 18.43 & 34.17 & 11.54 & 8.29 & 8.22 & 6.12 & 382.43 & 565.52 & 245.36 & 348.29 & 370.56 \\
\hline 182 & 460 & 50.28 & 7.83 & 5.5 & 36.12 & 12.08 & 8.6 & 8.73 & 6.71 & 145.77 & 256.05 & 44.96 & 148.65 & 133.39 \\
\hline 183 & 549 & 54.54 & 8.3 & 5.42 & 32.9 & 10.88 & 8.67 & 7.39 & 5.96 & 130.72 & 224.45 & 104.72 & 99.54 & 94.15 \\
\hline 184 & 357 & 47.53 & 10.35 & 6.33 & 33.68 & 10.79 & 9.31 & 7.66 & 5.92 & 58.46 & 107.89 & 39.57 & 42.44 & 43.93 \\
\hline 185 & 369 & 50.98 & 8.3 & 7.04 & 33.65 & 10.78 & 9.31 & 7.65 & 5.91 & 95.51 & 133.21 & 126.42 & 78.58 & 43.82 \\
\hline 186 & 28 & 8.58 & 17.21 & 2.63 & 32.08 & 10.35 & 9.02 & 7.36 & 5.35 & 6.01 & 9.77 & 4.92 & 6.12 & 3.23 \\
\hline 187 & 427 & 51.78 & 19.83 & 6.28 & 29.22 & 9.41 & 8.67 & 6.51 & 4.63 & 131.05 & 229.04 & 83.78 & 109.19 & 102.20 \\
\hline 188 & 474 & 64.33 & 17.49 & 8.73 & 29.75 & 9.59 & 8.71 & 6.63 & 4.82 & 186.42 & 211.25 & 213.37 & 170.84 & 150.21 \\
\hline 189 & 56.3 & 13.3 & 48.84 & 3.14 & 32.4 & 10.48 & 8.96 & 7.39 & 5.57 & 12.17 & 20.05 & 11.15 & 8.16 & 9.32 \\
\hline 190 & 15 & 8.74 & 56.7 & 5.09 & 32.23 & 10.63 & 8.67 & 7.42 & 5.51 & 3.85 & 6.87 & 2.57 & 3.39 & 2.58 \\
\hline 191 & 68.4 & 17.71 & 35.58 & 4.59 & 33.86 & 10.83 & 9.28 & 7.89 & 5.86 & 38.60 & 41.48 & 38.73 & 36.10 & 38.08 \\
\hline 192 & 274 & 67.14 & 18.32 & 16.45 & 33.99 & 11 & 8.98 & 8.07 & 5.94 & 18.77 & 32.45 & 20.71 & 11.68 & 10.25 \\
\hline 193 & 827 & 142.8 & 11.9 & 24.66 & 31.69 & 10.29 & 8.44 & 7.44 & 5.52 & 131.01 & 224.51 & 102.01 & 109.10 & 88.42 \\
\hline 194 & 239 & 56.11 & 7.66 & 13.17 & 30.94 & 9.91 & 8.52 & 6.95 & 5.56 & 36.60 & 60.50 & 38.82 & 25.94 & 21.12 \\
\hline 195 & 87.8 & 27.61 & 4.4 & 8.68 & 38.49 & 11.08 & 13.52 & 7.99 & 5.9 & 42.01 & 48.10 & 59.27 & 40.08 & 20.58 \\
\hline 196 & 204 & 38.21 & 8.59 & 7.16 & 29.6 & 9.31 & 9.18 & 6.25 & 4.86 & 16.72 & 12.11 & 45.25 & 4.02 & 5.49 \\
\hline 197 & 690 & 80.15 & 5.98 & 9.31 & 33.78 & 10.13 & 11.29 & 7.01 & 5.35 & 151.76 & 172.39 & 248.13 & 126.59 & 59.93 \\
\hline 198 & 247 & 31.82 & 9.59 & 4.1 & 31.22 & 9.42 & 10.35 & 6.5 & 4.95 & 33.53 & 40.04 & 67.62 & 17.67 & 8.82 \\
\hline 199 & 128 & 14.64 & 5.83 & 1.67 & 33.71 & 9.55 & 12.12 & 6.74 & 5.3 & 39.88 & 49.44 & 69.83 & 26.50 & 13.73 \\
\hline 200 & 737 & 65.39 & 17.21 & 5.8 & 26.52 & 8.71 & 8.42 & 5.72 & 3.67 & 167.74 & 171.60 & 190.56 & 191.22 & 117.57 \\
\hline 201 & 694 & 64.77 & 15.15 & 6.04 & 24.34 & 8.27 & 7.96 & 4.91 & 3.2 & 36.13 & 32.52 & 76.12 & 33.88 & 1.99 \\
\hline 202 & 469 & 42.53 & 9.03 & 3.86 & 21.75 & 7.19 & 7.36 & 4.41 & 2.79 & 55.89 & 82.02 & 70.23 & 67.16 & 4.14 \\
\hline 203 & 8,561 & 305.23 & 7.08 & 10.88 & 22.83 & 7.73 & 7.24 & 4.82 & 3.04 & 393.40 & 138.11 & 772.10 & 532.16 & 131.23 \\
\hline 204 & 389 & 49.65 & 22.73 & 6.34 & 27.77 & 8.78 & 8.68 & 6.24 & 4.07 & 138.53 & 160.69 & 145.44 & 143.81 & 104.20 \\
\hline 205 & 126 & 35.51 & 26.7 & 10 & 27.57 & 9 & 8.58 & 6.13 & 3.86 & 35.89 & 45.72 & 37.83 & 36.81 & 23.21 \\
\hline 206 & 206 & 35.15 & 28.28 & 6 & 27.91 & 8.99 & 8.37 & 6.26 & 4.29 & 73.09 & 91.84 & 83.20 & 65.02 & 52.31 \\
\hline 207 & 95.6 & 17.33 & 41.84 & 3.14 & 28.53 & 9.53 & 8.06 & 6.33 & 4.61 & 41.66 & 67.94 & 40.21 & 28.77 & 29.73 \\
\hline 208 & 45 & 14.82 & 40.29 & 4.88 & 28.17 & 9.27 & 8.16 & 6.3 & 4.44 & 19.04 & 26.18 & 21.86 & 15.33 & 12.78 \\
\hline 209 & 3,429 & 140.26 & 13.22 & 5.74 & 26.18 & 8.85 & 7.59 & 5.8 & 3.94 & 149.03 & 235.03 & 200.14 & 100.73 & 60.21 \\
\hline 210 & 4,493 & 205.11 & 10.11 & 9.36 & 25.55 & 8.61 & 7.49 & 5.68 & 3.77 & 183.89 & 277.41 & 253.92 & 131.35 & 72.89 \\
\hline
\end{tabular}


Table 1. Basin characteristics for streamflow-gaging stations in or near Texas, with at least 8 years of mean annual

\begin{tabular}{|c|c|c|c|c|c|c|c|c|c|}
\hline $\begin{array}{c}\text { USGS } \\
\text { station } \\
\text { no. }\end{array}$ & $\begin{array}{c}\text { Map } \\
\text { no. } \\
\text { (pl. 1) }\end{array}$ & USGS station name & $\begin{array}{c}\text { Region } \\
\text { no. }\end{array}$ & $\begin{array}{c}\text { Base } \\
\text { station } \\
\text { no. }\end{array}$ & Latitude & Longitude & $\begin{array}{l}\text { First } \\
\text { year } \\
\text { of } \\
\text { record }\end{array}$ & $\begin{array}{l}\text { Last } \\
\text { year } \\
\text { of } \\
\text { record }\end{array}$ & $\begin{array}{c}\text { Weight } \\
\text { factor }\end{array}$ \\
\hline 08206700 & 211 & San Miguel Creek near Tilden, Texas & 6 & 08208000 & $28^{\circ} 35^{\prime} 14^{\prime \prime}$ & $98^{\circ} 32^{\prime} 44^{\prime \prime}$ & 1965 & 1990 & 26 \\
\hline 08207000 & 212 & Frio River at Calliham, Texas & 6 & 08205500 & $28^{\circ} 29^{\prime} 31^{\prime \prime}$ & $98^{\circ} 20^{\prime} 47^{\prime \prime}$ & 1961 & 1980 & 20 \\
\hline 08208000 & 213 & Atascosa River at Whitsett, Texas & 6 & & $28^{\circ} 37^{\prime} 18^{\prime \prime}$ & $98^{\circ} 17^{\prime} 02^{\prime \prime}$ & 1961 & 1990 & 30 \\
\hline 08211520 & 214 & Oso Creek at Corpus Christi, Texas & 6 & 08164000 & $27^{\circ} 42^{\prime} 40^{\prime \prime}$ & $97^{\circ} 30^{\prime} 06^{\prime \prime}$ & 1973 & 1990 & 18 \\
\hline 08212400 & 215 & Los Olmos Creek near Falfurrias, Texas & 6 & 08189500 & $27^{\circ} 15^{\prime} 51^{\prime \prime}$ & $98^{\circ} 08^{\prime} 08^{\prime \prime}$ & 1968 & 1983 & 16 \\
\hline 08374000 & 216 & Alamita Creek near Presidio, Texas & 2 & & $29^{\circ} 31^{\prime} 15^{\prime \prime}$ & $104^{\circ} 17^{\prime} 40^{\prime \prime}$ & 1961 & 1990 & 30 \\
\hline 08374500 & 217 & Terlingua Creek near Terlingua, Texas & 2 & & $29^{\circ} 12^{\prime} 00^{\prime \prime}$ & $103^{\circ} 36^{\prime} 15^{\prime \prime}$ & 1961 & 1990 & 30 \\
\hline 08405500 & 218 & Black River above Malaga, New Mexico & 2 & & $32^{\circ} 13^{\prime} 48^{\prime \prime}$ & $104^{\circ} 09^{\prime} 00^{\prime \prime}$ & 1961 & 1990 & 30 \\
\hline 08408500 & 219 & Delaware River near Red Bluff, New Mexico & 2 & & $32^{\circ} 01^{\prime} 12^{\prime \prime}$ & $104^{\circ} 03^{\prime} 00^{\prime \prime}$ & 1961 & 1990 & 30 \\
\hline 08431700 & 220 & Limpia Creek above Fort Davis, Texas & 2 & 08400000 & $30^{\circ} 36^{\prime} 48^{\prime \prime}$ & $104^{\circ} 00^{\prime} 04^{\prime \prime}$ & 1967 & 1986 & 20 \\
\hline 08431800 & 221 & Limpia Creek below Fort Davis, Texas & 2 & 08374000 & $30^{\circ} 40^{\prime} 52^{\prime \prime}$ & $103^{\circ} 47^{\prime} 30^{\prime \prime}$ & 1963 & 1977 & 15 \\
\hline 08433000 & 222 & Barrilla Draw near Saragosa, Texas & 2 & 08405500 & $30^{\circ} 57^{\prime} 28^{\prime \prime}$ & $103^{\circ} 27^{\prime} 33^{\prime \prime}$ & 1976 & 1983 & 8 \\
\hline 08435700 & 223 & Sunny Glen Canyon near Alpine, Texas & 2 & 08128000 & $30^{\circ} 22^{\prime} 52^{\prime \prime}$ & $103^{\circ} 44^{\prime} 08^{\prime \prime}$ & 1969 & 1977 & 9 \\
\hline 08435800 & 224 & Coyanosa Draw near Fort Stockton, Texas & 2 & 08374000 & $31^{\circ} 02^{\prime} 27^{\prime \prime}$ & $103^{\circ} 08^{\prime} 15^{\prime \prime}$ & 1965 & 1977 & 13 \\
\hline 08447020 & 225 & Independence Creek near Sheffield, Texas & 2 & 08128000 & $30^{\circ} 27^{\prime} 07^{\prime \prime}$ & $101^{\circ} 43^{\prime} 58^{\prime \prime}$ & 1975 & 1984 & 10 \\
\hline 08449000 & 226 & Devils River near Juno, Texas & 2 & 08449400 & $29^{\circ} 57^{\prime} 48^{\prime \prime}$ & $101^{\circ} 08^{\prime} 42^{\prime \prime}$ & 1964 & 1973 & 10 \\
\hline 08449400 & 227 & Devils River at Pafford Crossing near Comstock, Texas & 2 & & $29^{\circ} 40^{\prime} 35^{\prime \prime}$ & $101^{\circ} 00^{\prime} 00^{\prime \prime}$ & 1961 & 1990 & 30 \\
\hline 08455000 & 228 & Pinto Creek near Del Rio, Texas & 2 & 08195000 & $29^{\circ} 08^{\prime} 45^{\prime \prime}$ & $100^{\circ} 43^{\prime} 05^{\prime \prime}$ & 1961 & 1968 & 8 \\
\hline
\end{tabular}

${ }^{1}$ Station also was included in this region to enhance the analysis. 
streamflow data, 1961-90-Continued

\begin{tabular}{|c|c|c|c|c|c|c|c|c|c|c|c|c|c|c|}
\hline \multirow{2}{*}{$\begin{array}{c}\text { Map } \\
\text { no. } \\
\text { (pl. 1) }\end{array}$} & \multirow{2}{*}{$\begin{array}{l}\text { Contri- } \\
\text { buting } \\
\text { drainage } \\
\text { area } \\
(\text { A) } \\
\left(\mathrm{mi}^{2}\right)\end{array}$} & \multirow{2}{*}{$\begin{array}{c}\text { Main } \\
\text { stream } \\
\text { length } \\
(\mathrm{L}) \\
(\mathrm{mi})\end{array}$} & \multirow{2}{*}{$\begin{array}{c}\text { Stream } \\
\text { slope } \\
(\mathrm{SL}) \\
(\mathrm{ft} / \mathrm{mi})\end{array}$} & \multirow{2}{*}{$\begin{array}{c}\text { Basin } \\
\text { shape } \\
\text { factor } \\
\text { (SH) }\end{array}$} & \multicolumn{5}{|c|}{$\begin{array}{l}\text { Precipitation } \\
\quad \text { (in.) }\end{array}$} & \multicolumn{5}{|c|}{$\begin{array}{l}\text { Estimated 1961-90 streamflow } \\
\left(\mathrm{ft}^{3} / \mathrm{s}\right)\end{array}$} \\
\hline & & & & & Annual & Spring & $\begin{array}{l}\text { Sum- } \\
\text { mer }\end{array}$ & Fall & $\begin{array}{l}\text { Win- } \\
\text { ter }\end{array}$ & Annual & Spring & $\begin{array}{l}\text { Sum- } \\
\text { mer }\end{array}$ & Fall & $\begin{array}{l}\text { Win- } \\
\text { ter }\end{array}$ \\
\hline 211 & 783 & 85.18 & 8.05 & 9.27 & 25.58 & 8.69 & 7.56 & 5.66 & 3.67 & 56.33 & 103.32 & 67.86 & 38.85 & 15.29 \\
\hline 212 & 5,491 & 221.33 & 9.64 & 8.92 & 25.48 & 8.59 & 7.51 & 5.63 & 3.75 & 231.80 & 355.96 & 282.16 & 170.60 & 118.48 \\
\hline 213 & 1,171 & 92.21 & 6.33 & 7.26 & 27.26 & 9.16 & 8.02 & 5.86 & 4.22 & 115.56 & 201.95 & 132.18 & 73.23 & 54.89 \\
\hline 214 & 90.3 & 11.77 & 4.74 & 1.53 & 31.09 & 8.75 & 11.51 & 5.98 & 4.85 & 28.08 & 28.80 & 50.11 & 21.44 & 11.97 \\
\hline 215 & 476 & 64 & 11.18 & 8.6 & 23.42 & 7.88 & 8.03 & 4.18 & 3.33 & 5.62 & 4.27 & 16.83 & 0.59 & 0.78 \\
\hline 216 & 1,504 & 101.6 & 40.69 & 6.86 & 14.93 & 3.57 & 7.79 & 2.51 & 1.06 & 20.67 & 14.16 & 57.20 & 10.09 & 1.22 \\
\hline 217 & 1,070 & 98.53 & 29.97 & 9.07 & 17.1 & 4.1 & 8.85 & 2.8 & 1.35 & 63.99 & 66.56 & 156.07 & 29.53 & 3.80 \\
\hline 218 & 343 & 56.7 & 47.6 & 9.37 & 15.48 & 3.71 & 8.2 & 2.3 & 1.27 & 13.00 & 12.62 & 21.10 & 9.38 & 8.92 \\
\hline 219 & 689 & 60.2 & 39.5 & 5.26 & 13.45 & 3.1 & 7.1 & 2.1 & 1.15 & 10.45 & 9.05 & 20.88 & 8.52 & 3.34 \\
\hline 220 & 52.4 & 11.39 & 129.62 & 2.48 & 20.65 & 4.6 & 11.62 & 3.01 & 1.42 & 2.24 & 1.89 & 6.27 & 0.81 & 0.00 \\
\hline 221 & 227 & 31.03 & 70.63 & 4.24 & 18.38 & 4.17 & 10.19 & 2.73 & 1.29 & 3.73 & 2.69 & 10.27 & 1.49 & 0.47 \\
\hline 222 & 612 & 84.55 & 12.77 & 11.68 & 16.74 & 3.86 & 8.93 & 2.63 & 1.32 & 9.09 & 0.24 & 35.45 & 0.23 & 0.42 \\
\hline 223 & 29.7 & 8.07 & 119.52 & 2.19 & 18.85 & 4.3 & 10.43 & 2.81 & 1.31 & 0.41 & 0.00 & 1.17 & 0.45 & 0.00 \\
\hline 224 & 1,182 & 89.16 & 26.9 & 6.73 & 15.93 & 3.87 & 8.09 & 2.67 & 1.3 & 4.22 & 8.82 & 7.91 & 0.10 & 0.05 \\
\hline 225 & 763 & 62.35 & 27.1 & 5.1 & 19.92 & 6.31 & 6.65 & 4.34 & 2.62 & 13.31 & 13.69 & 9.71 & 18.78 & 11.06 \\
\hline 226 & 2,730 & 112.27 & 10.17 & 4.62 & 20.69 & 6.14 & 7.69 & 4.15 & 2.71 & 175.79 & 84.15 & 441.29 & 94.87 & 82.84 \\
\hline 227 & 3,961 & 146.02 & 9.95 & 5.38 & 20.9 & 6.35 & 7.72 & 4.15 & 2.68 & 365.11 & 263.06 & 606.69 & 354.06 & 236.63 \\
\hline 228 & 249 & 44.23 & 17.43 & 7.85 & 21.03 & 7.64 & 6.35 & 4.22 & 2.82 & 39.07 & 24.25 & 104.74 & 9.99 & 17.30 \\
\hline
\end{tabular}


Table 2. Regression equations for estimating mean annual and mean seasonal runoff for regions of Texas

$\left[\mathrm{mi}^{2}\right.$, square miles; in., inches; Q, mean runoff in cubic feet per second; ann, annual—calendar year; spr, spring—April, May, and June; sum, summer-July, August, and September; fall—October, November, and December; win, winter-January, February, and March; A, contributing drainage area in square miles; MAP, mean annual precipitation in inches; MSP, mean seasonal precipitation in inches; - , not applicable; +, analysis for this region also includes stations from other regions]

\begin{tabular}{|c|c|c|c|c|c|c|}
\hline \multirow{2}{*}{$\begin{array}{c}\text { Region } \\
\text { no. }\end{array}$} & \multirow{2}{*}{$\begin{array}{l}\text { Weighted least-squares } \\
\text { regression equation }\end{array}$} & \multicolumn{2}{|c|}{$\begin{array}{l}\text { Range of indicated independent } \\
\text { variables in corresponding region }\end{array}$} & \multirow{2}{*}{$\begin{array}{l}\text { Weighted } \\
\text { standard } \\
\text { error of } \\
\text { estimate } \\
\text { (percent) }\end{array}$} & \multirow{2}{*}{$\begin{array}{l}\text { Weighted } \\
\text { standard } \\
\text { error of } \\
\text { estimate } \\
\left(\log _{10} \text { units) }\right.\end{array}$} & \multirow{2}{*}{$\begin{array}{c}\text { No. of } \\
\text { stations in } \\
\text { analysis for } \\
\text { equation }\end{array}$} \\
\hline & & $\begin{array}{c}\text { Contributing } \\
\text { drainage area } \\
\left(\mathrm{mi}^{2}\right)\end{array}$ & $\begin{array}{l}\text { Precipitation } \\
\text { (in.) }\end{array}$ & & & \\
\hline \multicolumn{7}{|l|}{1,2} \\
\hline & $\mathrm{Q}_{\mathrm{ann}}=10^{-6.048} \mathrm{~A}^{1.123} \mathrm{MAP}_{\mathrm{ann}}{ }^{3.293}$ & & 13.45 to 22.18 & 77 & 0.2954 & 24 \\
\hline & $\mathrm{Q}_{\mathrm{spr}}=10^{-3.403} \mathrm{~A}^{1.135} \mathrm{MSP}_{\mathrm{spr}}^{2.055}$ & & 3.10 to 8.65 & 112 & .3911 & 24 \\
\hline & $\mathrm{Q}_{\text {sum }}=10^{-5.781} \mathrm{~A}^{1.307} \mathrm{MSP}_{\text {sum }} 3.939$ & 29.7 to 3,961 & 6.35 to 11.62 & 118 & .4041 & 24 \\
\hline & $\mathrm{Q}_{\text {fall }}=10^{-3.281} \mathrm{~A}^{1.092} \mathrm{MSP}_{\text {fall }}{ }^{2.561}$ & & 1.90 to 4.34 & 213 & .5686 & 24 \\
\hline & $\mathrm{Q}_{\text {win }}=10^{-3.805} \mathrm{~A}^{1.248} \mathrm{MSP}_{\text {win }} 3.225$ & & 1.06 to 2.82 & 282 & .6434 & 24 \\
\hline \multicolumn{7}{|l|}{3} \\
\hline & $\mathrm{Q}_{\mathrm{ann}}=10^{-4.240} \mathrm{~A}^{0.885} \mathrm{MAP}_{\mathrm{ann}} 2.470$ & & 18.43 to 31.75 & 47 & .1928 & 34 \\
\hline & $\mathrm{Q}_{\mathrm{spr}}=10^{-3.196} \mathrm{~A}^{0.894} \mathrm{MSP}_{\mathrm{spr}} 2.783$ & & 5.91 to 10.95 & 54 & .2183 & 34 \\
\hline & $\mathrm{Q}_{\text {sum }}=10^{-4.439} \mathrm{~A}^{0.870} \mathrm{MSP}_{\text {sum }} 4.066$ & 11.8 to 5,972 & 7.06 to 8.82 & 53 & .2164 & 34 \\
\hline & $\mathrm{Q}_{\text {fall }}=10^{-2.255} \mathrm{~A}^{0.968} \mathrm{MSP}_{\text {fall }} 1.666$ & & 2.81 to 7.05 & 62 & .2474 & 34 \\
\hline & $\mathrm{Q}_{\text {win }}=10^{-3.002} \mathrm{~A}^{1.005} \mathrm{MSP}_{\text {win }} 2.824$ & & 1.82 to 5.52 & 49 & .2016 & 34 \\
\hline \multicolumn{7}{|l|}{4} \\
\hline & $\mathrm{Q}_{\mathrm{ann}}=10^{-8.285} \mathrm{~A}^{0.913} \mathrm{MAP}_{\mathrm{ann}} 5.300$ & & 18.83 to 30.66 & 56 & .2272 & 22 \\
\hline & $\mathrm{Q}_{\mathrm{spr}}=10^{-5.894} \mathrm{~A}^{0.920} \mathrm{MSP}_{\mathrm{spr}} 5.572$ & & 5.74 to 10.43 & 53 & .2152 & 22 \\
\hline & $\mathrm{Q}_{\text {sum }}=10^{-0.925} \mathrm{~A}^{0.895}$ & 33.3 to 6,160 & - & 73 & .2843 & 22 \\
\hline & $\mathrm{Q}_{\text {fall }}=10^{-4.007} \mathrm{~A}^{1.013} \mathrm{MSP}_{\text {fall }}{ }^{3.808}$ & & 3.41 to 7.13 & 87 & .3251 & 22 \\
\hline & $\mathrm{Q}_{\text {win }}=10^{-3.269} \mathrm{~A}^{0.909} \mathrm{MSP}_{\text {win }} 3.906$ & & 2.25 to 5.87 & 73 & .2829 & 22 \\
\hline \multicolumn{7}{|l|}{5} \\
\hline & $\mathrm{Q}_{\mathrm{ann}}=10^{-0.211} \mathrm{~A}^{0.846}$ & & - & 58 & .2322 & 27 \\
\hline & $\mathrm{Q}_{\mathrm{spr}}=10^{-3.131} \mathrm{~A}^{0.884} \mathrm{MSP}_{\mathrm{spr}} 3.004$ & & 8.27 to 11.16 & 60 & .2416 & 27 \\
\hline & $\mathrm{Q}_{\text {sum }}=10^{-0.669} \mathrm{~A}^{1.019}$ & 8.24 to 1,315 & - & 62 & .2466 & 27 \\
\hline & $\mathrm{Q}_{\text {fall }}=10^{-0.257} \mathrm{~A}^{0.849}$ & & - & 64 & .2543 & 27 \\
\hline & $\mathrm{Q}_{\text {win }}=10^{-2.826} \mathrm{~A}^{0.915} \mathrm{MSP}_{\text {win }} 3.412$ & & 3.20 to 6.12 & 120 & .4115 & 27 \\
\hline \multicolumn{7}{|l|}{$6+$} \\
\hline & $\mathrm{Q}_{\mathrm{ann}}=10^{-6.320} \mathrm{~A}^{0.834} \mathrm{MAP}_{\mathrm{ann}}{ }^{4.013}$ & & 21.75 to 38.61 & 54 & .2183 & 23 \\
\hline & $\mathrm{Q}_{\mathrm{spr}}=10^{-4.700} \mathrm{~A}^{0.747} \mathrm{MSP}_{\mathrm{spr}}^{4.759}$ & & 7.19 to 11.83 & 71 & .2761 & 23 \\
\hline & $\mathrm{Q}_{\text {sum }}=10^{-3.569} \mathrm{~A}^{0.860} \mathrm{MSP}_{\text {sum }} 3.344$ & 28.0 to 8,561 & 7.24 to 13.52 & 44 & .1842 & 23 \\
\hline & $\mathrm{Q}_{\text {fall }}=10^{-3.756} \mathrm{~A}^{0.804} \mathrm{MSP}_{\text {fall }}^{4.061}$ & & 4.18 to 8.39 & 107 & .3790 & 23 \\
\hline & $\mathrm{Q}_{\text {win }}=10^{-4.382} \mathrm{~A}^{0.976} \mathrm{MSP}_{\text {win }}^{4.757}$ & & 2.79 to 5.96 & 68 & .2666 & 23 \\
\hline
\end{tabular}


Table 2. Regression equations for estimating mean annual and mean seasonal runoff for regions of TexasContinued

\begin{tabular}{|c|c|c|c|c|c|c|}
\hline \multirow{2}{*}{$\begin{array}{l}\text { Region } \\
\text { no. }\end{array}$} & \multirow{2}{*}{$\begin{array}{l}\text { Weighted least-squares } \\
\text { regression equation }\end{array}$} & \multicolumn{2}{|c|}{$\begin{array}{l}\text { Range of indicated independent } \\
\text { variables in corresponding region }\end{array}$} & \multirow{2}{*}{$\begin{array}{c}\text { Weighted } \\
\text { standard } \\
\text { error of } \\
\text { estimate } \\
\text { (percent) }\end{array}$} & \multirow{2}{*}{$\begin{array}{l}\text { Weighted } \\
\text { standard } \\
\text { error of } \\
\text { estimate } \\
\text { ( } \log _{10} \text { units) }\end{array}$} & \multirow{2}{*}{$\begin{array}{c}\text { No. of } \\
\text { stations in } \\
\text { analysis fol } \\
\text { equation }\end{array}$} \\
\hline & & $\begin{array}{c}\text { Contributing } \\
\text { drainage area } \\
\left(\mathrm{mi}^{2}\right)\end{array}$ & $\begin{array}{l}\text { Precipitation } \\
\quad \text { (in.) }\end{array}$ & & & \\
\hline \multicolumn{7}{|l|}{7} \\
\hline & $\mathrm{Q}_{\mathrm{ann}}=10^{-7.585} \mathrm{~A}^{0.997} \mathrm{MAP}_{\mathrm{ann}} 4.609$ & & 30.95 to 42.97 & 33 & 0.1394 & 22 \\
\hline & $\mathrm{Q}_{\mathrm{spr}}=10^{-4.546} \mathrm{~A}^{0.944} \mathrm{MSP}_{\mathrm{spr}}{ }^{4.184}$ & & 10.82 to 14.17 & 31 & .1334 & 22 \\
\hline & $\mathrm{Q}_{\text {sum }}=10^{-4.750} \mathrm{~A}^{0.943} \mathrm{MSP}_{\text {sum }} 4.337$ & 26.0 to 572 & 7.33 to 9.77 & 60 & .2412 & 22 \\
\hline & $\mathrm{Q}_{\text {fall }}=10^{-3.879} \mathrm{~A}^{1.048} \mathrm{MSP}_{\text {fall }}^{3.523}$ & & 6.67 to 11.09 & 58 & .2321 & 22 \\
\hline & $\mathrm{Q}_{\text {win }}=10^{-3.390} \mathrm{~A}^{1.061} \mathrm{MSP}_{\text {win }} 3.343$ & & 5.68 to 9.35 & 42 & .1760 & 22 \\
\hline \multicolumn{7}{|l|}{$8+$} \\
\hline & $\mathrm{Q}_{\mathrm{ann}}=10^{-3.628} \mathrm{~A}^{0.803} \mathrm{MAP}_{\mathrm{ann}}^{2.337}$ & & 24.97 to 41.27 & 24 & .1046 & 24 \\
\hline & $\mathrm{Q}_{\mathrm{spr}}=10^{-2.463} \mathrm{~A}^{0.785} \mathrm{MSP}_{\mathrm{spr}}^{2.576}$ & & 9.93 to 12.63 & 24 & .1032 & 24 \\
\hline & $\mathrm{Q}_{\text {sum }}=10^{0.279} \mathrm{~A}^{0.773}$ & 23 to 1,240 & - & 31 & .1318 & 24 \\
\hline & $\mathrm{Q}_{\text {fall }}=10^{-2.223} \mathrm{~A}^{0.812} \mathrm{MSP}_{\text {fall }}{ }^{2.198}$ & & 6.58 to 11.09 & 34 & .1434 & 24 \\
\hline & $\mathrm{Q}_{\text {win }}=10^{-1.509} \mathrm{~A}^{0.819} \mathrm{MSP}_{\text {win }} 1.788$ & & 5.73 to 9.24 & 25 & .1089 & 24 \\
\hline \multicolumn{7}{|l|}{9} \\
\hline & $\mathrm{Q}_{\mathrm{ann}}=10^{-8.742} \mathrm{~A}^{1.086} \mathrm{MAP}_{\mathrm{ann}} 5.180$ & & 29.60 to 43.28 & 31 & .1300 & 24 \\
\hline & $\mathrm{Q}_{\mathrm{spr}}=10^{-6.136} \mathrm{~A}^{1.013} \mathrm{MSP}_{\mathrm{spr}}^{5.50}$ & & 9.31 to 12.75 & 36 & .1518 & 24 \\
\hline & $\mathrm{Q}_{\text {sum }}=10^{-4.723} \mathrm{~A}^{1.135} \mathrm{MSP}_{\text {sum }} 3.860$ & 17.3 to 838 & 8.29 to 13.52 & 44 & .1832 & 24 \\
\hline & $\mathrm{Q}_{\text {fall }}=10^{-4.934} \mathrm{~A}^{1.048} \mathrm{MSP}_{\text {fall }}{ }^{4.664}$ & & 6.25 to 10.19 & 53 & .2168 & 24 \\
\hline & $\mathrm{Q}_{\text {win }}=10^{-4.491} \mathrm{~A}^{1.074} \mathrm{MSP}_{\text {win }} 4.547$ & & 4.86 to 8.69 & 54 & .2184 & 24 \\
\hline \multicolumn{7}{|l|}{10} \\
\hline & $\mathrm{Q}_{\mathrm{ann}}=10^{-3.079} \mathrm{~A}^{1.066} \mathrm{MAP}_{\mathrm{ann}} 1.701$ & & 41.40 to 50.66 & 18 & .0787 & 24 \\
\hline & $\mathrm{Q}_{\mathrm{spr}}=10^{-0.213} \mathrm{~A}^{1.104}$ & & - & 21 & .0889 & 24 \\
\hline & $\mathrm{Q}_{\text {sum }}=10^{-3.485} \mathrm{~A}^{1.051} \mathrm{MSP}_{\text {sum }} 2.651$ & 23.4 to 1,365 & 7.70 to 11.37 & 40 & .1665 & 24 \\
\hline & $\mathrm{Q}_{\text {fall }}=10^{-0.281} \mathrm{~A}^{1.046}$ & & - & 31 & .1307 & 24 \\
\hline & $\mathrm{Q}_{\mathrm{win}}=10^{-1.478} \mathrm{~A}^{1.033} \mathrm{MSP}_{\text {win }} 1.440$ & & 9.29 to 12.61 & 21 & .0892 & 24 \\
\hline \multicolumn{7}{|l|}{11} \\
\hline & $\mathrm{Q}_{\mathrm{ann}}=10^{-3.616} \mathrm{~A}^{0.942} \mathrm{MAP}_{\mathrm{ann}}^{2.166}$ & & 40.25 to 56.64 & 21 & .0895 & 29 \\
\hline & $\mathrm{Q}_{\mathrm{spr}}=10^{-1.731} \mathrm{~A}^{0.938} \mathrm{MSP}_{\mathrm{spr}}{ }^{1.653}$ & & 11.26 to 15.40 & 21 & .0883 & 29 \\
\hline & $\mathrm{Q}_{\text {sum }}=10^{-4.829} \mathrm{~A}^{1.002} \mathrm{MSP}_{\text {sum }}{ }^{4.145}$ & 8.65 to 3,486 & 9.54 to 15.04 & 39 & .1620 & 29 \\
\hline & $\mathrm{Q}_{\text {fall }}=10^{-1.512} \mathrm{~A}^{0.905} \mathrm{MSP}_{\text {fall }} 1.417$ & & 10.38 to 14.30 & 31 & .1311 & 29 \\
\hline & $\mathrm{Q}_{\text {win }}=10^{-1.959} \mathrm{~A}^{0.973} \mathrm{MSP}_{\text {win }}{ }^{1.989}$ & & 8.15 to 13.41 & 17 & .0716 & 29 \\
\hline
\end{tabular}

\footnotetext{
${ }^{1}$ Mean annual or mean seasonal precipitation for corresponding equation.
} 


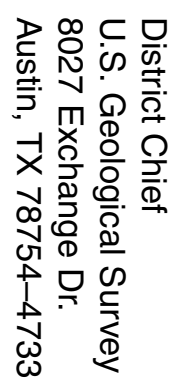

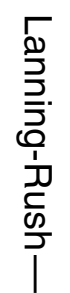

